\title{
Abscisic Acid Metabolism in Leaves and Roots of Four Vitis Species in Response to Water Deficit
}

Haley S. Toups ( $\sim$ htoups@nevada.unr.edu )

University of Nevada Reno

Noe Cochetel

University of Nevada Reno

Kevin Galdamez

University of Nevada Reno

Laurent Deluc

Oregon State University

Grant R. Cramer

University of Nevada Reno

\section{Research Article}

Keywords: ABA, ABA metabolism, leaves vs. roots, NCED3, Vitis

Posted Date: March 15th, 2021

DOI: https://doi.org/10.21203/rs.3.rs-289346/v1

License: (c) (1) This work is licensed under a Creative Commons Attribution 4.0 International License. Read Full License 


\section{Abstract}

Background: Abscisic acid is a phytohormone involved in water deficit response. Abscisic acid metabolism is regulated by biosynthesis, conjugation, and catabolism. NCED3 is the rate limiting step of abscisic acid biosynthesis and is a key contributor to plant water deficit responses. In this study NCED3 transcript accumulation and abscisic acid metabolism were further characterized as key water deficit responses in four Vitis species (Vitis vinifera (Cabernet Sauvignon), Vitis champinii (Ramsey), Vitis riparia (Riparia Gloire), and Vitis vinifera x Vitis girdiana (SC2)) under three levels of water deficit in leaves and roots.

Results: The concentrations of abscisic acid and derivative metabolites increased with water deficit and was dependent upon the species. RNA-Seq and RT-qPCR data were consistent with the changes in abscisic acid metabolite concentrations; the corresponding transcript abundances substantiate NCED3 as a key gene in the water deficit response; however, NCED3 protein concentrations assayed in Western Blots were not affected. Major differences in abscisic acid metabolism at the gene, protein, and metabolite levels were detected between leaves and roots in these four species. NCED3 transcript abundance and abscisic acid concentration in drought-tolerant Ramsey increased earlier and more significantly than the other species during long-term, moderate to severe water deficits but were not stimulated as much by short-term, rapid dehydration. In drought-sensitive Riparia, NCED3 transcript abundance and abscisic acid metabolite concentrations increased to a lower extent than in Ramsey during moderate to severe water deficits, but short-term rapid dehydration induced a significantly higher abscisic acid concentration in Riparia than Ramsey.

Conclusions: Grapevine species have distinct abscisic acid metabolism that depends highly on the severity and duration of stress and organ (leaves or roots). This study confirms that abscisic acid metabolism and NCED3 are part of a core water deficit response in Vitis species. Relative quantities of transcripts, proteins, abscisic acid and derivative metabolites were determined, but many aspects of abscisic acid metabolism and water deficit responses warrant additional investigation. This study provides a better understanding of how Vitis is adapted to dry environments, which may be exploited for future breeding programs.

\section{Background}

Water deficit (WD) and drought are natural climactic events that occur all across the world [1] and may become more frequent and severe in the near future [2-4]. For sessile crops, the consequences of severe WD can be devastating. Historically, droughts decreased global crop production by $10 \%$ [5]. Plants evolved many mechanisms to respond to, withstand or avoid, and ultimately survive water scarcity. To illicit drought tolerance, an intricate and complex WD response signals throughout the plant involving multiple steps of regulation. For example, epigenetic modification and chromatin remodeling allow easier accessibility to dehydration responsive genes like RESPONSIVE TO DESICCATION 29A (RD29A) [6, 7]. Transcriptionally, genes may be transcribed (or not) [8], alternatively spliced [9], and present alternative 
untranslated region (UTR) isoforms [10,11] or polyadenylation [12]. MicroRNAs like miR160 target growth related auxin response factor transcripts like $A R F 10$ for degradation $[13,14]$. siRNAs are another mechanism of regulation that contribute to WD response [15]. Post-translational modifications can mark enzymes for degradation or activation [16-18] like the phosphorylation of SNRK2s, which are critical proteins in the abscisic acid (ABA) signaling pathway [19]. The numerous steps of regulation ultimately lead to the proteins and metabolites that result in the phenotype of the plant. Understanding WD response in plants continues to expand, but few studies investigate WD response at more than one level of regulation. This work provides a more comprehensive view of core ABA metabolism in response to WD by examining transcripts, protein, and metabolites in four distinct Vitis species at three levels of WD in leaves and roots.

$A B A$ is a phytohormone associated with abiotic stress response including WD. ABA signaling is initiated when ABA is perceived by the PYRABACTIN RESISTANCE1/PYR1-LIKE/REGULATORY COMPONENTS OF ABA RECEPTORS (PYR/PYL/RCAR) receptors [20-23] that then interact with PP2Cs allowing for the activation of SNRK2s $[24,25]$. SNRK2s activate ABA responsive transcription factors (TFs) including AREBs [26, 27], ABFs [19, 28-30], ABIs [31, 32], and many others. This core ABA signaling pathway activates the transcription of numerous downstream targets including aquaporins [33-35], suberin TFs $[36,37]$, galactinol synthases $[38,39]$, and $\mathrm{RDs}[40,41]$ that ultimately result in physiological changes that better allow a plant to survive under adverse conditions.

ABA metabolism consists of three pathways: biosynthesis, conjugation, and catabolism. Like cytokinin [42], gibberellins [43], brassinosteroids [44], and strigolactones [45], ABA is synthesized from isoprenoid products of the methylerythritol phosphate (MEP) pathway in the plastid [46]. Phytoene synthase condenses two geranylgeranyl-diphosphate (GGPP) molecules into the C40 carotene, phytoene $[47,48]$. Through a series of desaturation [49-51] and cyclization reactions [52]; ß-carotene is formed and hydroxylated (B-CAROTENE HYDROXYLASE (BETA-OHASE 2)) into zeaxanthin [53, 54]. Zeaxanthin is epoxidated (ZEAXANTHIN EPOXIDASE (ZEP/ABA DEFICIENT 1 (ABA1))) into violaxanthin through intermediary antheraxanthin [55-57]. Neoxanthin, synthesized by NEOXANTHIN SYNTHASE (NXS), and violaxanthin participate in the rate limiting step of ABA biosynthesis performed by NINE-CISEPOXYCAROTENOID DIOXYGENASE (NCED) [58-60]. The resulting molecule, xanthoxin is transported out of the plastid into the cytoplasm where ABA biosynthesis is completed [61]. XANTHOXIN DEHYDROGENASE (ABA DEFICIENT 2 (ABA2)) protonates the epoxy group of xanthoxin to form ABA aldehyde [62]. Finally, ABA aldehyde is oxidized to active ABA by ABA ALDEHYDE OXIDASE (AAO) [63] with a molybdenum cofactor sulfurase (ABA DEFICIENT 3 (ABA3)).

ABA may be conjugated with UDP-glucose [64] or amino acids [65] to generate an inactive storage form of $A B A$ [66]. ABA glucose ester (ABA-GE) is the most predominant storage form of $A B A$ [67]. ABA-GE is formed by the esterification of ABA with UDP-glucose by UDP-glucose glucosyltransferase (UGT) $[68,69]$. Once conjugated, ABA-GE is stored in the vacuole [70-72]. ABA-GE is readily de-esterified to active ABA via $ß$-d-glucosidases (BG or $B G L U$ ) $[73,74]$. This pathway is thought to be important for quick $A B A$ release and response as compared to ABA biosynthesis [74-76]. 
ABA catabolism is composed of three conduits through ABA hydroxylation to form 7', 8', or 9'-hydroxy ABA [77]. ABA hydroxylation is performed by ABA hydroxylases (CYTOCHROME P450 (CYP707A)) [78]. $A B A$ catabolites function as signaling molecules affecting physiology, development, and environmental adaptation $[79,80]$, but the distinct role of each catabolite remains unresolved. 7'-hydroxy ABA (7'OH $A B A$ ) is hydroxylated at the C-7' methyl group. 8'-hydroxy ABA is isomerized in to phaseic acid (PA) [78] and further reduced to dihydrophaseic acid (DPA) [81]. Neophaseic acid (NeoPA) is formed from 9'hydroxy ABA and is thought to be a minor catabolite. Catabolites may be conjugated [82] or further degraded.

ABA appears to be a key regulator of physiological and transcriptional responses to water deficit $[8,83$, 84]. Previously, RNA sequencing analysis (RNA-Seq) was performed on four diverse Vitis species (Vitis vinifera cv. Cabernet Sauvignon clone 8 (CS), Vitis champinii cv. Ramsey (RA), Vitis riparia cv. Riparia Gloire (RI), and Vitis vinifera $x$ Vitis girdiana hybrid SC2 (SC)) in response to one and two weeks (W1 and W2) of well-watered (Control) and a natural dry-down water deficit (WD) conditions [8]. This experiment paired with an additional one-week severe WD experiment, in which these four grapevines experienced a stress more severe than the stress level achieved after two-weeks of moderate WD (based on stem water potential values) [8]. These species differ in drought tolerance with RA appearing the most drought tolerant and RI the most susceptible [8]. RA retained the highest stomatal conductance, photosynthesis, and stem water potential of the four species under both long-term WD treatments [8]. RI was the first species to shut down photosynthesis and experience stress levels resulting in leaf loss or even vine death in response to moderate and severe long-term WD [8]. Specific and shared transcriptomic responses between the four Vitis species were identified [8]. The transcript abundance of many abscisic acid signaling genes were strongly increased by WD with distinct differences between the species in both experiments. A weighted gene co-expression network analysis (WGCNA) identified a water-deficit core gene set with the ABA biosynthesis gene, NCED3, as a potential hub in all four species. RA emerged as the most physiologically active and transcriptionally responsive species following WD. However, ABA and ABA derived metabolite quantification was not presented in the previous study.

In this study, prior observations of these four grapevine species' responses to WD and ABA metabolism [8] were built upon with the addition of $A B A$ and $A B A$ metabolite quantification as well as additional WD experiments. The term ABA metabolite refers to the group of compounds quantified in this study that are derived from ABA: ABA-GE, PA, DPA, NeoPA, and 7'OH ABA. Although there were numerous differences between the four species, the contrast of drought tolerant RA to susceptible RI will be the focus of the results presented here. It was expected $A B A$ metabolite quantification would mirror the transcript abundance of $A B A$ metabolism genes in response to WD, which proved generally true. The ABA concentrations and transcriptional responses presented here are coherent with other studies that investigate $A B A$ metabolism in grapevine leaves and roots $[85,86]$. Additionally, differences in ABA metabolism at the transcript and metabolite levels are associated to physiological responses and differences in drought tolerance and sensitive cultivars of wheat [87], rice [88], and soybean [89]. More drought tolerant plant cultivars have higher rates of photosynthesis [89], reduced reactive oxygen species $[88,90]$, and higher stem water potentials [87] like what is observed for RA [8]. Additionally, ABA 
concentrations are shown to vary between organ, duration of water deficit, and between drought sensitive and tolerant wheat cultivars [87], as was found in this study with grapevines.

In this study, increased ABA metabolism was confirmed as a key response to WD through quantitative analysis of ABA, ABA metabolites, ABA metabolism transcripts (RNA-Seq and RT-qPCR), and NCED3 protein (Western Blots) from three experiments in which the species were exposed to varying intensity and duration of WD. It was hypothesized NCED3 transcripts, NCED3 protein, and ABA concentrations were correlated with each other. The relationship between NCED3, NCED3, and ABA is not well described for grapevine nor other plant species. Commonly, transcript abundance is expected to explain protein abundance. However, this scenario is found to be the exception, not the rule [91-95]. In this study, NCED3 protein did not increase like NCED3 transcript abundance and ABA concentration in leaves and roots in these grapevine species under these WD conditions. The goals of this work were to further establish ABA biosynthesis as a core response to WD in Vitis, examine ABA metabolism in leaves and roots, and compare the ABA metabolisms of the four Vitis species that differ in drought tolerance.

\section{Results}

For the moderate and severe long-term WD experiments previously described [8], CS, RA, RI, and SC vines were subjected to one or two week of moderate or severe water deficit, or well-watered daily. Vines were grown in pots under greenhouse conditions. At W1 and W2 of treatment various measurements were taken [8]. Leaves and roots were harvested after W1 and W2 of treatment. The one-week severe water deficit treatment reached a more acute level of stress (lower stem water potentials) than the stress level obtained after two-weeks of moderate water deficit (see Methods).

\section{Grapevine organs and species differ in $A B A$ and $A B A$ metabolite concentrations during one and two-week moderate WD}

Leaf and root $A B A$ and $A B A$ metabolite concentrations are different between the four species after a one and two-week moderate WD treatment. ABA, ABA-GE, PA, DPA, NeoPA, and 7'OH ABA were extracted and quantified (Figs. 1 and 2) as previously described [96]. There were significant differences between treatments, organs, species, and weeks for all metabolite concentrations (pmol.mg DW ${ }^{-1}$; DW refers to dry weight) except for 7'OH ABA. The term "significant" will be used in this work to mean statistically significant at a p-value of 0.05 or less. To simplify comparisons to WD, metabolite quantities of the Controls of all species were grouped together per organ per week (Figs. 1 and 2) and were referred to as the average of the Controls (Avg Ctrl).

Grapevine organs had different [ABA] under Control and WD treatments. There was a significant difference in [ABA] between W1 WD leaves and roots, between W2 Control leaves and roots, and between W2 WD leaves and roots. Per organ per week only RA WD W1 leaves [ABA] was significantly increased 
relative to that of Avg Ctrl in (Fig. 1). WD RA W1 had at least 3-fold higher average [ABA] in the leaves and roots than WD RI W1. At W2, the [ABA] for all species was significantly higher in WD leaves relative to Avg Ctrl leaves, and WD RA had a significantly higher [ABA] than WD SC (Fig. 1). W2 roots also had significantly higher [ABA] for all WD species than those of Avg Ctrl (Fig. 1), and again WD RA had an average [ABA] 2-fold and 3-fold higher than WD RI in the leaves and roots, respectively. However, unlike the leaves there was no significant difference between the species in the roots treated with WD at W2 (Fig. 1). As a general rule, [ABA] can be ranked by species during moderate WD as RA $>C S>R I>S C$ in both leaves and roots.

The concentrations of the storage form of $A B A, A B A-G E$, were significantly higher than [ABA] in leaves and lower than [ABA] in the roots (Fig. 1) and in all combinations per treatment per week. At W2, RA and SC WD leaves had significantly higher [ABA-GE] than Avg Ctrl, and WD RA leaves had significantly higher [ABA-GE] than WD RI leaves (Fig. 1). In the roots, RA was the only species to have significantly higher [ABA-GE] than Avg Ctrl, but WD RA W2 roots were only significantly different from those of CS (Fig. 1).

In general, ABA catabolism was enhanced by WD. At W1, WD [ABA catabolite] were similar to respective Controls (Fig. 2). W1 WD [DPA] in WD RA roots were significantly higher than WD RI roots. By W2, [ABA catabolite] increased by 2 - to 10 -fold in both leaves and roots relative to W1. Like ABA, ABA catabolites were generally higher in WD W2 leaves than WD W2 roots but were at comparable levels in leaves and roots at W1 (Fig. 2). For example, WD W2 RA had > 10-fold increase in [PA] in leaves relative to roots. In WD W2 leaves, RA was the only species that had significantly different concentrations of all four ABA catabolites relative to Avg Ctrl (Fig. 2). RA and CS WD W2 roots generally had higher concentrations of all four quantified catabolites than those of the other two species (Fig. 2), but there was no significant difference between the WD species for any catabolite in the roots at W2 (Fig. 2). Although not always significant, each species displayed unique responses to moderate WD, particularly in W2, via changes in [ABA catabolite] with RA (and CS) generally having higher [ABA catabolite] than the other species in both organs, consistent with the higher [ABA] observed in these species (Fig. 1).

There were clear differences in the use of the catabolite pathways in grapevines. The major catabolic pathway was through 8'OH ABA with [DPA] and [PA] being much higher than [7'OH ABA] at W1 and W2 (Fig. 2). NeoPA, which also goes through the 8'OH ABA catabolism pathway, had very low concentrations relative to the other catabolites (Fig. 2). Nevertheless, these concentrations also increased with [ABA] induced by moderate WD with RA $>\mathrm{CS}>\mathrm{Rl}$; $\mathrm{SC}$ had very low concentrations and showed no increase due to WD (Fig. 2). PA, an intermediate in the $8^{\prime} \mathrm{OH} A B A$ catabolic pathway had much higher concentrations than DPA in WD leaves. However, the reverse was true in WD roots, where [DPA] was higher than [PA].

To further understand $A B A$ metabolism in the plant as a whole, the distribution of $A B A$ and $A B A$ metabolites was examined for the whole plant (the sum of leaf and root total $A B A$ and $A B A$ metabolites, to be defined as Total ABA Metabolites). ABA and all ABA metabolites were summed to estimate the total ABA produced by the plant (Additional File 1A) or per organ (Additional File 1B). On a whole plant basis, there were significant increases in [Total ABA Metabolite] by W2 (Additional File 1A) providing evidence 
for an increase in ABA biosynthesis along with other metabolism pathways. Total ABA Metabolite distribution in W1 was less clear and may indicate that changes in ABA metabolite concentrations were the result of redistribution of $A B A$ (e.g. conjugation, catabolism and transport). [ABA] and each [ABA metabolite] per organ was also divided by the summed Total ABA Metabolite concentration (Additional File $1 \mathrm{~A}$ ) to determine the distribution of each ABA metabolite (Additional File 2). This approach revealed ABA-GE represented the major portion of the leaf $A B A$ metabolites in W1 Controls $(0.38 \pm 0.05)$, followed by PA $(0.23 \pm 0.03), A B A(0.17 \pm 0.03)$, and DPA $(0.13 \pm 0.02)$ (Additional File 2$)$. However, RA WD W1 leaves had a significant decrease in the proportion of ABA-GE from $0.48 \pm 0.15$ to an average $0.20 \pm 0.08$, indicating $A B A$ metabolism was shifted from $A B A-G E$ to other $A B A$ metabolites such as $A B A$ and DPA. The reduction of ABA-GE pools was specific to W1 RA leaves; there was no significant difference in RA Control vs. WD W2 leaves indicating this shift in ABA metabolism was an early response to WD (Additional File 2). The other species did not show the same shift in ABA-GE distribution in WD leaves. CS ABA-GE $(0.2 \pm 0.02)$ remained constant in $W 1$ and $W 2$ and did not change significantly in response to WD in the leaves (Additional File 2). Likewise, the proportion of ABA-GE in WD W2 roots decreased relative to Control in all of the species except for $C S$, which again was proportionately low relative to the other three species (Additional File 2). No such changes were observed in WD W1 roots. ABA and ABA-GE in the roots in general were a much smaller proportion of Total ABA Metabolites than DPA.

These grapevine species have different relative $A B A$ and $A B A$ metabolite ratios. To further evaluate the range of $[A B A]$ and [ABA metabolite] between the species, the WD:C ratios of [ABA] and [ABA metabolite] were investigated (Fig. 3). ABA and 7'OH ABA had the highest z-scores of all the metabolites quantified (Fig. 3). Such changes were not obvious for 7'OH ABA in Total ABA Metabolites (Additional File 2). RA W1 leaves had the highest score for ABA (Fig. 3). RI W1 leaves had the highest score for WD:C ABA-GE (Fig. 3), indicating ABA-GE may have been accumulating in RI W1 WD leaves. The WD:C ABA-GE score for RA W1 leaves was about two STD lower than the average WD:C ABA metabolite average (Fig. 3), indicating ABA-GE may be deconjugated into active ABA and/or downstream catabolites, supporting similar observations from Additional File 2. ABA-GE also had a negative score for the roots of all species in W1 and W2 (Fig. 3), possibly reflecting ABA activation through this pathway.

The ABA catabolites have different scores in the different species and organs. 7'OH ABA had the highest scores for RA W1 roots and leaves and RA and CS W2 roots and leaves (Fig. 3) despite 7'OH ABA having lower concentrations than metabolites representing catabolism through 8'OH ABA (PA and DPA). The high z-scores for 7'OH ABA in these select species and organs may indicate these species redistribute a higher portion of $A B A$ catabolism through the 7'OH ABA pathway than the other species in response to WD, but this pathway either catabolizes lower [ABA] than that through 8'OH ABA, PA, and DPA (Fig. 2) or catabolites are further degraded into compounds not quantified here. Alternatively, the high z-scores for $77^{\prime} \mathrm{OH}$ ABA may be attributed to the large difference between W2 root average RA and CS Control [7' $\mathrm{OH}$ $\mathrm{ABA}]\left(0.14 \pm 0.03 \mathrm{pmol} \cdot \mathrm{mg} \mathrm{DW}^{-1}\right)$ and average WD RA and CS $\left(5.31 \pm 1.67 \mathrm{pmol} \cdot \mathrm{mg} \mathrm{DW}^{-1}\right)$ that result in a greater fold difference (38-fold difference) than those of PA (6-fold difference) and DPA (3-fold difference); this difference is reflected in the z-score despite differences in [catabolite]. RA W1 roots and 
RA W2 leaves had the highest score for NeoPA and DPA, which were also about one STD above the average ABA metabolite ratio (Fig. 3). RA was the only species to have a positive score for PA and DPA in W2 leaves (Fig. 3). The differences between the catabolites indicate ABA may be preferentially deactivated by different mechanisms in each species in each organ and with increasing WD stress. For example, at W2, RA was the only species to have positive scores in leaves and roots for PA ( 0.5 STD from mean), while all other species had negative scores. Combined with the positive scores for RA W2 leaves and roots 7'OH ABA and RA W2 leaves NeoPA scores, RA is clearly catabolizing ABA relative to the other species. WD RA W2 [7'OH ABA] has the greatest change relative to Control [ $\left.7^{\prime} \mathrm{OH} A B A\right]$ and the average [ABA metabolite]. NeoPA, PA, and DPA had the highest z-scores in W1 across various organs and species, but the scores were either lower or negative for W2 indicating these catabolism pathways may be favored at different times during WD. At W1, RA and SC had positive scores for DPA in the roots, but not the leaves, hinting these catabolism pathways may be utilized differently across organs. The different species, organs, and times have different scores for the ABA metabolites.

\section{One and two-week moderate WD significantly increases ABA biosynthesis, conjugation, and catabolism transcripts abundance}

ABA metabolism gene transcript abundance significantly increase in response to WD. Differences in many $A B A$ metabolite gene transcript abundances mirrored [ABA metabolite] and may partially explain differences observed between species. Previously, the ABA biosynthesis gene, NCED3, was identified as a potential hub gene in response to WD in this W1 and W2 moderate WD experiment [8]. Additional works indicate NCED3 as a potential WD hub gene [97]. To support this hypothesis, the RNA-Seq series, PRJNA516950, was analyzed with the more accurate CS clone 8 v1.0 reference genome [98] as opposed to the PN40024 reference genome [99] as was done previously [8]. The goal of this analysis was to investigate the transcript abundance of ABA metabolism genes in response to WD. CS ABA metabolism genes were identified with protein basic local alignment search tool (BLAST) using known ABA metabolism PN40024 protein sequences as a query. Orthologs were confirmed based on the highest total score, E-value, and length. When two BLAST hits were highly similar for the same query, the hits were identified as alleles of the same gene. In total, 46 ABA metabolism genes were identified from the primary (alternative (alt)1) and/or secondary (alt2) CS haplotig sequences (Additional File 3). Alleles are referred to as alternatives without a designated haplotype because the phased chromosome-scale assembly was not complete for the CS clone 8 v1.0 genome. Detailed explanation of alt assignment is in Additional File 3. In total, allele(s) for three NCED genes (NCED3, NCED5 alt1 and 2, and NCED6 alt1 and 2) were identified in CS (Additional File 3). Both alleles were identified for BETA-OHASE 2, ABA ALDEHYDE OXIDASE 3 (AAO3), ABA1, ABA2, ABA3, BETA-1, 3-GLUCANASE 1 (BG1), ATP-BINDING CASSETTE 625 (ABCG25), and ATP-BINDING CASSETTE G22-LIKE (ABCG22-like). Several BGLU, BG, UGT, and CYP707A paralogs were also identified. Only one $A B C G 40$ and $N P Q 1$ (Violaxanthin de-epoxidase or NONPHOTOCHEMICAL QUENCHING 1) allele were annotated in this version of the CS genome. 
Organs showed significant differences in the genes expressed and gene expression levels (Additional File 4) [8]. There were significant differences in ABA metabolism genes comparing WD to Control in all four species per organ (Additional File 4). CS (4 and 0 genes in leaves and roots, respectively), RA (1 and 1 genes), and RI ( 1 and 0 genes) had the most differentially expressed ABA metabolism genes out of all WD vs. Control W1 contrasts (Additional File 4). RA W2 leaves and roots had the most differentially expressed ABA metabolism genes (21 and 17 genes in leaves and roots, respectively) in response to WD followed by CS (10 and 12 genes), SC (10 and 7 genes), and RI (4 and 8 genes) (Additional File 4). The transcripts of NCED3 (Fig. 4) and at least one putative BETA-OHASE 2 allele were significantly increased in response to WD in W2 leaves and roots of all four species (Additional File 4). However, the other differentially expressed ABA metabolism genes varied between species and organs.

RA has distinct ABA metabolism differentially expressed genes (DEGs) compared to the other species. In the previous analysis of this experiment, RA stood out transcriptionally and physiologically by outperforming the other species, having higher photosynthesis and greater and earlier transcriptomic responsiveness to WD [8]. To better understand the role each pathway of ABA metabolism (biosynthesis, conjugation, or catabolism) contributes to the transcriptomic responsiveness of RA and the other species, differential expression analyses (DEAs) contrasting WD to Control per time point per organ were focused upon using the 46 CS ABA metabolism genes. To identify specific genes differentially expressed in WD RA leaves and roots relative to WD organs from the other three species, DEA was performed as previously described [8] using RA WD contrasts (Additional File 5). The majority of WD responsive DEGs related to ABA metabolism in RA and the other species in either leaves or roots were involved in biosynthesis and conjugation (Additional File 5).

In this section, ABA metabolism DEGs that were unique to WD RA (relative to the WD of all three other species) will be discussed in order of ABA metabolism pathway (biosynthesis, (de)conjugation, and catabolism) sequentially in W1 leaves then roots and finally in W2 leaves followed by W2 roots. No gene was significantly differentially expressed in RA relative to all three of the other species in WD W1 leaves. NCED3, NCED5 alt2, and ABCG25 alt1 had significantly higher transcript abundance in RA WD W1 roots than those of the other species (Figs. 4 and 5 and Additional File 5). NCED3 was the only ABA metabolism DEG in RA W1 WD roots relative to Control (Additional File 4), but NCED3 was not a DEG in W1 roots of any other species at W1 in response to WD (Fig. 4 and Additional File 4). BG2 alt1 and UGT71C3 alt1 were also differentially expressed in RA W1 WD roots relative to the other three species, but these genes were not DEGs W1 root WD vs. Control contrast for any species.

There were nine ABA metabolism DEGs in RA leaves after W2 of WD relative to those of the other three species that were also DEGs in RA WD W2 leaves relative to RA Control W2 leaves (Figs. 4 and 6 and Additional Files 5 and 6). Four of these genes were involved in ABA biosynthesis: NCED3 alt1, NPQ1 alt1, $A B A 1$ alt1, $A A O 3$ alt1. NCED3 had significantly higher transcript abundance in RA WD W2 leaves than those of all other species (3.7-, 9.8-, and 6-fold difference for CS, RI, and SC respectively) (Fig. 4 and Additional File 5). NCED3 had the highest expression level of the five annotated NCEDs in both leaves and roots in W1 and W2 of WD in all species with the highest level of expression in RA (Fig. 4). The other 
NCEDs were lowly expressed (Fig. 4). NCED5 alternatives had higher expression in CS, while both NCED6 alternatives had higher expression levels in RA after W2 of WD compared to the other species (Fig. 4). NCED3 was also the ABA metabolism DEG that had the highest average TPM over all WD species and times compared to the other ABA metabolism DEGs that were significantly differentially expressed in RA WD W2 leaves vs. those of the other species and in RA Control vs. RA WD W2 leaves. Overall, NCED3 appears to be the major NCED contributing transcripts to downstream ABA biosynthesis. NPQ1 alt1 was significantly 4-fold lower in RA WD W2 leaves than those of the other species (Additional Files 5 and 6). $A B A 1$ alt1 was also significantly lower in RA WD W2 leaves than the WD W2 leaves of the three other species (Additional Files 5 and 6). Overall, average $A B A 1$ alt 1 transcript abundance over all treatments, times, and species was significantly higher in the leaves than the roots. $A A O 3$ alt 1 had significantly lower transcript abundance in RA than CS (16-fold difference), RI, and SC (both about 56-fold difference) (Additional Files 5 and 6) in W2 WD leaves. Although lowly expressed, average $A A O 3$ alt 1 transcript abundance was significantly higher in roots than that in leaves across all species, times, and treatments.

There were four ABA metabolism DEGs in RA leaves after W2 of WD that were also DEGs in W2 RA leaves in response to WD involved in deconjugation. The deconjugation DEGs were $B G 1$ alt1, $B G 1$ alt2, $B G 3$ alt1, and $B G 3$ alt2. $B G 1$ alt1 transcript abundance was significantly lower in RA WD W2 leaves than CS (6.5fold difference), RI, (26-fold difference), and SC (64-fold difference) (Fig. 5 and Additional Files 5). BG1 alt1 and alt2 were DEGs in RA WD leaves and roots relative to respective Controls, but neither $B G 1$ allele was a DEG in the organs of any other species (Additional File 2). BG1 alt1 had higher transcript abundance in roots than in leaves across all species, treatments, and times. BG1 alt2 also had significantly higher average transcript abundance in all Control and WD species roots relative to all Control and WD species leaves. In WD W2 roots, $B G 1$ transcripts alt1 and alt2 were the only significantly differentially expressed ABA metabolism DEGs in RA WD vs. the other three species WD contrasts with 16-40-fold lower abundance in RA than the other species (Fig. 5 and Additional File 5). Control RA and SC had the highest $B G 3$ alt1 expression in the leaves and roots in W2, but RA WD W2 had the lowest $B G 3$ alt1 expression in leaves and roots. Interestingly, per treatment, $B G 3$ alt1 transcript abundance was comparable in the leaves and roots for each species. $B G 3$ alt2 had the lowest transcript abundance in RA for all treatments and organs in W2 relative to the other species. CYP707A1 alt1 was the only ABA catabolism gene significantly differently expressed between RA and all three other species in W2 WD leaf contrasts and Control vs. WD contrasts (Additional Files 4-6). CYP707A1 alt1 had significantly higher transcript abundance in RA WD W2 leaves than those of the other three species (Additional Files 4 and 6 ), and like $A A O 3$ alt 1 and $B G 1, C Y P 707 A 1$ alt 1 average transcript abundance was higher in roots than leaves as an average over all species, treatments, and times.

\section{One and two-week moderate WD significantly increases ABA transport gene transcript abundance}

ABA transport genes were significantly differentially expressed in response to WD. ABA transporter genes $A B C G 25$ alt 1 and alt2 and $A B C G 40$ had significantly higher average transcript abundance in roots than leaves in all species, weeks, and treatments. Neither $A B C G 25$ alt2 nor $A B C G 40$ were higher in CS, RA, and 
RI WD W2 roots or RA WD W2 leaves relative to respective Control (Fig. 6 and Additional File 4). On average $A B C G 25$ alt1 transcript abundance was 4-fold higher in WD than Control W2 leaf and root samples (Additional File 4). ABCG25 alt2 transcripts were significantly higher in RA WD W2 leaves ( 32fold increase) and RA WD W2 roots ( 8-fold increase) relative to respective Control (Fig. 6 and Additional File 5).

RA had significantly increased $A B C G 25$ and $A B C G 40$ transcript abundance relative to other species after W2 of WD in leaves and roots (Additional File 5). ABCG25 alt1 was significantly ( 3-fold) higher in RA than CS WD W2 leaves, and about 4-fold change higher in RA W1 and W2 roots than those of the other three species. $A B C G 25$ alt2 transcript abundance was also significantly higher in RA WD W2 leaves than those of RI and SC (Additional File 5). After W1 of WD, RA roots had 8-fold change increase in $A B C G 40$ transcripts relative to those of CS, but $A B C G 40$ was not significantly different in Control vs. WD contrasts for either RA or CS in W1 (Additional File 4).

\section{ABA metabolite gene transcript abundance may partially explain ABA metabolite concentrations}

Multiple ABA metabolism genes had significantly increased transcript abundance similar to the increases observed in ABA metabolites. To more easily compare species response to WD and link [ABA metabolite] to upstream transcripts, the average ratio of WD:C transformed TPM were expressed as a z-score per ABA metabolism gene group (e.g. NCED3, NCED5 alt1 and 2, and NCED6 alt1 and 2 are included in the NCED gene group) with darker colors indicating greater difference from the mean ratio of the ABA metabolism genes (Fig. 7). RA clearly stands out in this comparison, having the highest score for $B$-carotene hydroxylases (roots W1 and W2), zeaxanthin epoxidases (roots W1 and W2), NCEDs (leaves and roots W1 and W2), AAO3s (leaves W1), UDP-glucose glucosyl transferases (leaves and roots W2), ß-d-glucosidases (roots W1), and various $A B A$ hydroxylases (leaves $\mathrm{W} 1$ and roots $\mathrm{W} 1$ and $\mathrm{W} 2$ ) (Fig. 7) that may partially explain the high [ABA] and [ABA metabolite] observed in RA. RA also had the lowest scores for violaxanthin de-epoxidases (leaves W1), xanthoxin dehydrogenases (roots W2), ß-d-glucosidases (leaves W2), and $A B A$ aldehyde oxidases (leaves and roots week 2) (Fig. 7). The low score ( -2 STDs from the mean) and low expression (Fig. 7) of B-d-glucosidases as well as the high z-score for UDP-glucose glucosyl transferases ( 1.75 STD from mean) in RA W2 leaves may explain the high [ABA-GE] observed in RA WD W2 leaves; ABA may be conjugated into ABA-GE but not deconjugated allowing [ABA-GE] to increase. The higher [ABA-GE] in RI W1 WD leaves relative to the other species (Fig. 7) and the higher score for $B$ - $d$-glucosidase ( 2 STD from mean) may indicate ABA-GE is an important source of ABA for $\mathrm{RI}$ in earlier WD response. RI and SC had the lowest [catabolite] in the leaves, which is paralleled in the score of the $A B A$ hydroxylases.

\section{ABA metabolism genes were correlated with multiple WGCNA modules}

WGCNA was performed on leaves and root samples separately with ABA and the ABA metabolites as additional traits (Figs. 8 and 9 and Additional File 7). Gene association to each module was calculated 
between each gene and the eigengene of each module [100] (Additional File 7). ABA metabolism genes were spread across multiple gene modules. In the leaves, 30 WGCNA modules were identified (Fig. 8). WD was positively correlated with five modules. These modules included lightyellow, darkgreen, brown, saddlebrown, and green (Fig. 8). Generally, the ABA metabolites were positively correlated with the same five modules as WD (Fig. 8). The 46 ABA metabolism genes were spread across 20 different modules in leaves. However, within these modules only lightyellow overlapped with WD, $A B A$, and the ABA metabolites. The lightyellow module contained the greatest number of ABA metabolism genes (seven) including NCED3 (0.91), NCED6 alt1 (0.77), BETA-OHASE 2 alt1 (0.74), NCED6 alt2 (0.70), BETA-OHASE 2 alt2 (0.64), CYP707A4 alt1_1 (0.62), and UGT73B4 alt1 (0.61) (Additional File 3).

NCED3 was clearly a hub gene in the lightyellow module, being the 15th most correlated gene to the eigengene representing this module. The top 20 genes of a module are considered hub genes. Hub genes are highly connected to all other genes in the module. Disruption of hub genes disturbs the gene expression of numerous other genes in a module. NCED3 was closely connected to ABA signaling genes in this module including the top 1 gene, HIGHLY ABA INDUCED 1 (HA/1) (VvCabSauv08_P0061F.ver1.0.g440640). Other top genes in the lightyellow module included RASRELATED PROTEIN 18 (RAB18) (VvCabSauv08_H0004F_076.ver1.0.g050030; top 9), HOMEOBOX-7 (HB-7 (VvCabSauv08_P0060F.ver1.0.g439740; top 12), and PP2C-8 (VvCabSauv08_P0452F.ver1.0.g610510; top 14) (Additional File 7). The position of NCED3 in the lightyellow module top genes far exceeded that of any other any ABA metabolism gene in any module; the second highest ranking gene was UGT71C4 alt2 (top 90) in the red module (Additional File 7). In the lightyellow module, NCED6 alt1, was the second occurring ABA metabolism gene in the top 259 genes (Additional File 7).

In the roots, 34 WGCNA modules were identified (Fig. 9). In total, ten modules were positively associated with WD (Fig. 9). Among the WD positively correlated modules, six were also positively correlated with 7'OH ABA, ABA, ABA-GE DPA, NeoPA, and PA (Fig. 9 and Additional File 7). Common positively correlated modules between the metabolites included royalblue, lightgreen, midnightblue, pink, and paleturquoise. The ABA metabolism genes were spread across 20 modules in the roots. The midnightblue module contained the greatest number of ABA metabolism genes (ten) including NCED3 (0.95), CYP707A2 alt1 (0.88), BETA-OHASE 2 alt1 (0.85), NCED5 alt2 (0.80), CYP707A3 alt1 (0.80), CYP707A4 alt1_1 (0.78), BETA-OHASE 2 alt2 (0.76), ABCG25 alt1 (0.76), NCED5 alt1 (0.75), and NCED6 alt1 (0.65) (Additional File $3)$. Of all ABA metabolism genes and all modules, NCED3 was the only hub gene corresponding to the midnightblue module (top 19) (Additional File 7) with $B G 3$ alt1 in the blue module (top 51) being the closest following ABA metabolism gene when considering all modules (Additional File 3 ). In the midnightblue module, CYP707A2 alt1 (top 191) was the next occurring ABA metabolism gene (Additional File 7). Like in the leaves, NCED3 was closely connected to ABA signaling hub genes in the midnightblue module in the roots; these genes included: RD26 (VvCabSauv08_H0024F_036.ver1.0.g115900; top 1), SEVEN IN ABSENTIA OF ARABIDOPSIS 2 (SINAT2) (VvCabSauv08_P0027F.ver1.0.g381860; top 6), NAC DOMAIN CONTAINING PROTEIN 47 (NACO47) (VvCabSauv08_P0024F.ver1.0.g368680; top 7), GALACTINOL SYNTHASE 1 (GolS1) (VvCabSauv08_P0018F.ver1.0.g349170 and 
VvCabSauv08_P0018F.ver1.0.g349150; top 9 and 10, respectively), and Hordeum vulgare L. 22 (HVA22E) (VvCabSauv08_P0095F.ver1.0.g483690, top 16) (Additional File 7).

Genes in the ABA metabolite and WD modules have roles in response to stress and stimulus. Gene ontology enrichment was performed using biological process terms for the brown, green, and lightyellow modules in the leaves and the midnightblue and pink modules in the roots (Additional File 8). These modules were selected for high correlation to WD and the ABA metabolites as well as for the number of ABA metabolism genes contained in each module. The gene ontology (GO) term corresponding to response to endogenous stimulus was enriched in all these modules (Additional File 8). All of these modules were enriched for the response to stress and response to stimulus GO terms (Additional File 8). All modules except green leaf included the response to abiotic stimuli GO term (Additional File 8). The lightyellow leaf module was enriched for the biosynthetic process $\mathrm{GO}$ term (Additional File 8), and lightyellow had the highest correlation with BETA-OHASE 2, NCED3, and NCED6 in the leaves (Additional File 3) supporting this grouping. The green leaf module was enriched for the catabolic process $\mathrm{GO}$ term (Additional File 8) and was most correlated with CYP707A1 alt1 (Additional File 3). The ABA metabolism gene functions were enriched with $\mathrm{GO}$ terms assigned to the modules most correlated with WD and the [ABA metabolite] supporting a link between transcript abundance and [metabolite].

\section{One-week severe WD significantly increases $A B A$ biosynthesis transcripts and metabolites, but does not change NCED3 protein}

A second experiment was performed with a more severe WD over W1 described previously [8]. Briefly, the same four Vitis species underwent a natural dry-down over the course of a week that achieved a stem water potential lower than that of vines that experienced the two-week moderate WD treatment. RT-qPCR was reported previously, and NCED3 NRQ were significantly increased in response to WD in all species except for RI; RA WD and CS WD had the highest NCED3 NRQ in the leaves and roots [8]. Fold difference (FD) of NCED3 protein abundance was quantified from western blots that were made relative to a CS Control leaf sample run on every gel as an inter-run caliber (IRC) as previously described [101] (Additional File 9). There was no significant difference of relative NCED3 between the treatments or species (Additional File 10). However, CS Control leaves and RA Control and WD leaves had significantly higher relative NCED3 protein than respective roots (Additional File 10).

ABA was quantified, and the [ABA] after W1 of severe WD were comparable to those of W2 moderate WD (Figs. 1 and 10). All WD treated leaves, except those of RI were significantly different from respective Control (Fig. 10), which was paralleled in NCED3 NRQ [8]. RA WD leaves had a significantly higher [ABA] than those of RI and SC, which was also observed in NCED3 NRQ. In the roots, no WD treated species had a significant difference from respective [ABA] Control or to other WD treated species (Fig. 10). The similarity in [ABA] between the W2 moderate and one-week severe WD experiments indicated that $A B A$ metabolism was dependent on severity and duration of WD stress. 


\section{Exposing leaves to short-term WD with increasing severity significantly increases NCED3 transcripts and ABA concentration, but it does not impact NCED3 protein abundance}

A WD time course experiment was conducted to examine NCED3 transcript abundance, relative NCED3 protein abundance, and $[A B A]$ in response to rapid dehydration WD. As all vines from previous experiments were submitted to the same treatments for the same long-term duration, there may have been a limited range of responses revealed in the previous experiments. To address the limited range of WD and long-term duration in the previous experiments, a third experiment was performed to expose leaves of three of the species (CS, RA, and RI) to a short-term time course of rapid dehydration WD. CS, $\mathrm{RA}$, and RI leaves underwent rapid dehydration or continual petiole irrigation under controlled conditions for $2,4,8$, and 24 hours.

Over the course of the experiment WD leaves had significantly lower stem water potential and lost significantly more water than Control leaves (Additional File 11). RI WD leaves had significantly lower water content at 2 hours of rapid dehydration relative to respective Control leaves, and CS and RA WD leaves had a significant change in water content by 8 hours of treatment, but there was not a significant difference between WD species at any timepoint. Both stomatal conductance (Gs) and photosynthesis (Ps) were significantly reduced over the course of the WD treatment (Additional File 11). Stem water potential, osmotic potential, and calculated turgor pressure [102] were also significantly reduced in the WD treated leaves (Additional File 11). There were significant differences between treatments and species for most physiological measurements, but there was not a significant difference for time in most cases (Additional File 11). For this reason, Control and WD physiological measurements were shown as an average of all species and time points (Additional File 11). Per time point per measurement there was no significant difference between WD species, but all WD species were significantly different from Control by two hours of treatment.

Each species increased NCED3 transcript abundance in response to the rapid dehydration (Fig. 11). There were no significant differences in NCED3 transcript abundance between the Control leaves of the species for any time point. NCED3 transcript abundance was significantly higher in CS rapid dehydration leaves at all time points relative to CS Control leaves with a general trend of NCED3 transcripts increasing with rapid dehydration time (Fig. 11 and Additional File 12). NCED3 transcript abundance was significantly higher in RA rapid dehydration leaves at all time points except 24 hours relative to RA Control leaves, but NCED3 NRQ was much lower in RA WD leaves than CS WD leaves (Fig. 11 and Additional File 12). Average RA NCED3 transcript abundance was highest after two hours of rapid dehydration and decreased over time, but RA rapid dehydration NCED3 transcripts at two hours of treatment were not significantly different than any other time point of rapid dehydration (Fig. 11 and Additional File 12). Surprisingly, NCED3 transcripts stayed constant in RI rapid dehydration leaves, and RI WD was only significantly different from RI Control after two hours of treatment (Fig. 11 and Additional File 12). Interestingly, there 
were no significant differences in NCED3 transcript abundance between the rapid dehydration leaves of the species per time point (Fig. 11 and Additional File 12).

[NCED3 protein] was not significantly different for any species in Control or rapid dehydration at any time point (Additional File 10). Western blots were performed as in the one-week severe water deficit experiment using a CS Control two-hour sample as an IRC [101]. RI rapid dehydration two- and four-hour leaves had the greatest variability in NCED3 protein sample between replicates. The NCED3 relative abundance similarity between Control and WD leaves in the rapid dehydration was comparable to those observed in the one-week severe WD.

[ABA] increased in response to short term rapid dehydration WD (Fig. 11). There was no significant difference in Control [ABA] between the species at any timepoint (Fig. 11 and Additional File 12). [ABA] in CS WD 24 hours was significantly different from RA WD 24 hours, but this was the only significant difference between WD species at any time (Fig. 11 and Additional File 12). CS WD [ABA] was significantly different from that of CS Control at two and 24 hours of treatment (Fig. 11 and Additional File 12). CS WD experienced a general increase in ABA with time like CS WD NCED3 transcript abundance (Fig. 11). RA WD had the lowest [ABA] of the WD treated species at all times; RA WD was only significantly different from RA Control after 24 hours of treatment (Fig. 11 and Additional File 12). RA [ABA] paralleled RA NCED3 transcript abundances (Fig. 11), which surprisingly did not increase as much as CS during this short-term WD treatment. RI WD had the highest [ABA] of the WD species at four and eight hours of treatment and steadily increased with time (Fig. 11 and Additional File 12). However, RI WD [ABA] did not follow the same trend as RI WD NCED3 transcript abundance (Fig. 11), which remained relatively constant throughout the stress. This observation may indicate $\mathrm{RI}$ is relying on a different source of ABA (like ABA-GE deconjugation) more than the other species under short-term rapid dehydration. Although the WD species were experiencing the same level and duration of stress and having similar physiological responses (Additional File 11), each species displayed unique ABA metabolism responses via NCED3 transcript abundance and [ABA] (Fig. 11 and Additional File 12). NCED3 transcript abundance and $[A B A]$ during short term rapid dehydration did not display the same responses as the longer-term moderate and severe WD, indicating ABA metabolism is highly dependent not only on organ and species but also on stress severity and duration.

\section{Discussion}

\section{Increases in NCED3 transcript abundance, ABA, and ABA metabolite concentrations were a core WD response in Vitis}

Overall, this work highlights differences in transcripts, a protein, and metabolites in the ABA metabolism pathway in two organs of four Vitis species in response to WD. Various [ABA] and [ABA metabolites] (Figs. 1-3 and 10-11) were in part explained by corresponding changes in upstream transcript abundances (Figs. 4-7, 11 and Additional File 7). Throughout these experiments, NCED3 stood out as a key WD response gene in both leaves and roots of all species, which was supported by WGCNA (Figs. 8- 
9), GO enrichment analysis (Additional File 8), and RT-qPCR (Fig. 11). From the WGCNA, the lightyellow module in the leaves and the midnightblue module in the roots contained the most ABA metabolism genes in each respective organ (Additional File 3). Both of these modules identified NCED3 as a hub gene (Additional File 7), meaning NCED3 was among the top 20 most connected genes to other genes in these modules. Not only was NCED3 a hub gene for WD and ABA metabolism associated modules in both the leaves and roots, but NCED3 was the only ABA metabolism hub gene (Additional File 7) of the 46 ABA metabolism genes. No other ABA metabolism gene was close to being a top gene in the lightyellow, midnightblue, or any other module (Additional File 7), further highlighting the importance of NCED3. NCED3 was closely connected to other ABA signaling genes in these modules. The lightyellow leaf and midnightblue root modules did not contain the same ABA metabolism genes, indicating a difference in ABA metabolism and signaling gene transcription in leaves and roots. Only BETA-OHASE2 alt1 and atl2, CYP707A4 alt1_1, NCED6 alt1, and NCED3 were in the lightyellow modules in the leaves and the midnight blue module in the roots. The commonality of NCED3 and other NCED genes correlating with modules associated to WD and corresponding to the most ABA metabolism genes in both organs support the hypothesis that NCED3 acts as a core gene in response to WD in both leaves and roots. In addition to the most ABA metabolism and signaling genes, the lightyellow and midnightblue modules include numerous genes involved in plastid function like plastid transkelotases and the chloroplastic $50 \mathrm{~S}$ ribosome subunit (Additional File 7). Other genes in these modules include aquaporins, ion transporters, ascorbate oxidase, galactinol synthase, and cysteine and sulfur regulatory genes, which have known associations to $A B A$ [38, 103-105] (Additional File 7). GO enrichment analysis further supported NCED3 as a hub gene with biosynthetic and abiotic stress response terms linked to the lightyellow and midnightblue modules. Additionally, NCED3 transcript abundance increased in all WD experiments described here regardless of severity, duration, organ, and even species (although there were distinct responses to each WD treatment for each organ and species). The increase in NCED3 transcripts and [ABA] detailed here from three WD experiments was reflected in downstream signaling and physiological responses (Additional File 11) [8]. Only one NCED3 allele was detected in the CS v1.0 and later in the CS v1.1 genome using available protein BLAST. However, a second allele (VvCabSauv08_v1_Primary000127F) was found with manual curation using only the alternative contig in the CS v1.1 genome. The two NCED3 alleles share $99 \%$ identity determined by Clustal Omega alignment, and therefore the transcript abundance for this gene was representative of both alleles.

\section{Leaves and roots differ in NCED3 transcript abundance, NCED3 protein levels, and ABA and ABA metabolite concentrations}

The primary site of ABA biosynthesis (leaves versus roots) as well as the initial site and signal to trigger a stress response during WD has been a controversial topic [106-108]. Recently, a small root sourced signaling peptide, CLE25, was identified in Arabidopsis thaliana that controls stomatal closure via ABA biosynthesis in the leaves [109]. However, there is no orthologous peptide in many species including 
grapevine, and the subtleties of WD detection in roots and shoots as well as the identities of longdistance signaling molecules (including ABA itself) remains elusive. These experiments characterize ABA metabolism in leaves and roots of grapevine. However, it is important to consider whole organs were used for these analyses. Specific cell types like the guard cell [110] or root endodermis [111] have specialized responses to $A B A$ and likely have unique regulation of NCED3 and ABA metabolism each of which require further investigation in these grapevines.

In these experiments, leaves and roots had different levels of ABA metabolism related transcripts (Figs. 4-7 and 11), NCED3 protein (Additional File 10), and ABA metabolites (Figs. 1-3 and 10-11). In both the moderate and severe WD experiments, leaves generally had higher abundance of NCED3 transcripts and [ABA] than roots. Leaves also had higher [NCED3 protein] than roots in the severe WD experiment. The differences in NCED3 transcripts, NCED3 protein, [ABA] and [ABA metabolite] between leaves and roots may reflect different sensitivity to $A B A$ between the organs. It is likely the [ABA] threshold to illicit a specific $A B A$ response is organ specific [86] and may involve $A B A$ transport between organs. Previously, exogenous application of $A B A$ was demonstrated to greatly affect transcriptomic signaling within berries, shoot tips, leaves, roots, and cell culture with no one gene demonstrating the same change in transcript abundance across all organs [86]. For example, ABCG22-like and ABI3 transcripts uniquely increased in the berries while MYB121 and DREB2H had specific transcript abundance accumulation in the roots. Organ specific sensitivity to other hormones has also been previously described. For example, exogenous $[\mu \mathrm{M}]$ of auxin stimulates leaf and shoot expansion but inhibits root elongation $[112,113]$. Gibberellins similarly demonstrated organ specificity with high concentrations biosynthesized in the stamen from which lower concentrations are transported out to support other floral organ development like the petals [114].

ABA, like gibberellins is biosynthesized [115-117] and transported throughout a plant [118]. ABA transport from vascular cells into guard cells is well documented with several identified transporters (ABCG40 [119], ABCG25 [120], ABCG22 [121], ABCG30, ABCG31 [122], and more recently NPF4.5 (AIT2) and NPF4.6 (AIT1) [123]). However, despite growing evidence that root ABA may be in part shoot sourced $[83,124,125]$, a pathway for $A B A$ transport into the root remains poorly described compared to that of ABA-vascular unloading and transport into guard cells [120] and endosperm-ABA unloading into the seed embryo [122]. In the moderate WD experiment, ABCG25 and ABCG40 had higher transcript abundance in roots than leaves for all species, treatments, and time points (Fig. 6), indicating possible ABA phloem unloading and $A B A$ transport into the roots. Literature further supports the possibility of shoot-sourced $A B A$ accumulating in the roots $[120,122,126]$. ABCG25 is predominantly expressed in phloem companion cells in Arabidopsis thaliana $[120,127]$, indicating ABA is transported down through the plant. ABCG25 transcripts accumulated in Arabidopsis thaliana roots in response to salt stress [126], and when nGFP was expressed under a $2.0 \mathrm{~kb}$ AtABCG25 promoter, a signal was observed in the root in the presence of $10 \mu \mathrm{M}$ ABA after 20 hours of treatment [128]. AtABCG40, responsible for importing ABA into guard cells, also plays a role in lateral root development and is expressed in primary and lateral roots in addition to being crucial for stomatal function [119]. 
ABA-GE is abundant during WD [82] and demonstrated to be the most abundant ABA metabolite in the moderate WD experiment (Fig. 1). However, ABA-GE transport regulation remains unresolved. Leaves had significantly higher [ABA-GE] than roots in the moderate WD experiment (Fig. 1), but the organs may have different requirements for this metabolite. BG1 had significantly higher transcript abundance in roots than leaves for the majority of species for both treatments and times in the moderate WD experiment (Fig. 5), indicating the possible importance of ABA-GE deconjugation in the roots. Despite low membrane permeability [129] and attempts to identify an ABA-GE transporter [119], this potential ABA transport pathway remains unsolved. ABA-GE transport may be one source of shoot derived $A B A$ in roots that has not been characterized [118]. ABA transport into the roots warrants further elucidation.

\section{Grapevine species differ in physiological and biochemical responses to WD}

In these experiments, species had distinct biochemical regulation of ABA. CS generally had the most variable physiological measurements [8], NCED3 transcript abundance, [ABA], and [ABA metabolite] in response to the various water deficit experiments. In all experiments, RI quickly closed stomata [8] and remained at this new homeostasis throughout the WD while having a small increase in ABA metabolite transcripts and [ABA] relative to CS and RA. SC demonstrated similar biochemical response to RI with lower transcript and metabolite levels. In the moderate and severe WD experiments, RA had higher [ABA] in both leaves and roots relative to the other species (Figs. 1-3 and 10). RA also had the highest NCED3 transcript abundance in moderate and severe WD treatments (Fig. 4) [8]. Additionally, RA had the highest levels of NCED3 transcripts (Fig. 4) and [ABA] (Fig. 1) at the earliest time point, indicating RA may be the most sensitive (earliest to respond) of the species investigated for long-term WD detection. RA maintained higher physiological function during the moderate and severe WD [8] despite higher levels of ABA than the other species (Figs. 1 and 10). These observations indicate RA may be less sensitive to [ABA] over time in terms of a physiological response (like stomatal closure) or RA may be enacting changes that better allow RA to function under WD (e.g. suberization or modified hydraulic conductance) relative to the other species. A number of aquaporin genes display differential and unique responses to water deficit in RA leaves (Additional File 4). Genes involved in cysteine biosynthesis and metabolism are also constitutively higher in the roots of RA [8]; thus, linking the gene expression and physiological responses to known ABA effectors that were not investigated here. The physiological and sensitive transcriptomic response that occurs at milder longer-term WD in RA indicates RA may be able to take advantage of moderate or longer-term WD by maintaining open stomata longer than the other species despite decreasing water availability. In the rapid dehydration WD, RA had lower [ABA] and NCED3 NRQ (Fig. 11) than the other species, but RA maintained the same stomatal conductance as the other species (Additional File 11), despite the increasing stress. The apparent stagnation of RA [ABA] until 24 hours of rapid dehydration represented the whole leaf and may not reflect [ABA] in specific cell types like the guard cells. RA may have different sensitivity to ABA and the sensitivity changes as well as changes in hydraulic signals over the duration of the stress. 
Drought tolerance is the ability of a vine to sustain physiological activity while minimizing or repairing damage during WD [130]. The drought tolerance of plants is associated with stomatal behaviors in response to WD and resultant use or preservation of available water. Grapevine responses to WD are characterized as belonging to a spectrum of stomatal reactions in the face of WD ranging from isohydric to anisohydric [131]. Isohydric species maintain a relatively constant leaf water potential through early stomatal closure during WD [132] like RI. Anisohydric species experience decreasing leaf water potential and maintain open stomata during WD [132] like RA. It is possible the iso- and aniso-hydric behaviors are controlled by different mechanisms (chemical and/or hydraulic regulation) altogether, by different mechanisms for different WD severities (moderate versus severe), or by different mechanisms at different time points (initial versus long-term) during a drought [132]. Isohydric behavior is often considered advantageous for conferring drought tolerance [131-134]. However, by the definition of drought tolerance [130], it may be worth reconsidering the association of isohydric grapevines with drought tolerance in favor of connection to drought avoidance. A vine that can maintain stomatal aperture (i.e. anisohydric), photosynthesis, and other physiological functions under decreasing water availability like RA may be considered more drought tolerant than a vine that shuts down early when experiencing stress like RI. Overall, ABA biosynthesis appeared to be a WD response that differentiated the species. The time frames investigated in these experiments emphasize the ABA metabolism and WD response of the different species depends on duration and severity of stress. WD experiments must be designed and compared to each other very carefully for this reason. More time points should be investigated in the future to better understand differences in short- and long-term WD and the transition between them in terms of ABA metabolism.

\section{The rapid dehydration response varies and is inconsistent with long-term slower dehydration responses}

Previously, a similar rapid dehydration experiment was performed [135]. The average water lost at two $(-0.139 \pm 0.022 \mathrm{~g})$ and four $(-0.122 \pm 0.012 \mathrm{~g})$ hours of rapid dehydration treatment lost were comparable to the previous experiment after two $(\sim-0.1 \mathrm{~g})$ and three $(\sim-0.14 \mathrm{~g})$ hours of a similar rapid dehydration treatment. However, in another similar rapid dehydration experiment where microarray transcript quantification was performed, but water loss was not quantified, NCED3 transcript abundance showed different expression patterns in CS, RA, and RI than were observed here [136]. These differences may be real or due to different transcriptomic technologies. Microarray data are often subject to crosshybridization of the probes and can be less reliable, such as the probe for NCED3 (VIT_19s0093g00550), which may cross-hybridize [137].The [ABA] curve of RI in the current rapid dehydration assay mirrored that of the NCED3 transcript curve that was previously observed and was expected in this experiment. The minor increase in RA and RI NCED3 transcript abundance at 24 hours of treatment was the most distinct difference between this and the previous experiment. In addition to the differences in technology, this distinction may be a result of differences in the age of leaves selected, the time of year, time of day, or even different experiences the plants had with WD in the past [138]. Control NCED3 transcript abundance was comparable for all species and time points between this and previous experiments. 


\section{NCED3 protein concentration was not changed by WD}

Despite differences in ABA biosynthesis transcripts and [ABA metabolite], [NCED3 protein] was generally constant for all species, treatments, and time points. Few studies consider NCED3 protein levels, although different Arabidopsis thaliana species have demonstrated similar NCED3 protein levels in response to 10 hours at low water potential, and differences in ABA accumulation were resultant of variations in protein sequence [139]. It is possible NCED3 protein sequence variation (Additional File 9) contributes to the observed differences in ABA accumulation in the species or NCED3 may have distinctive activity in the different species. In peanut, authors briefly mention increased ABA with a parallel increase in NCED3 protein [140], but this correlation was not observed in grapevine under these WD experiments. It is possible this relationship may be organ, stress, or time specific. While NCED3 protein was not found to increase with $A B A$ in these experiments, other proteins have been found to directly correlate with both NCED3 transcript abundance and [ABA] in plants. For example, overexpression of ATAF1 in Arabidopsis thaliana resulted in proportional increase in both NCED3 transcripts and ABA abundances in response to abiotic stress [141]. Another study in Arabidopsis thaliana examined transcript, [protein] and [metabolite] in response to illumination, which can be an abiotic stress, but NCED3 protein was not detected, indicating it was not an abundant protein under these conditions [142]. NCED3 activity has previously been screened [143], but a correlation between transcripts and protein was not made. Recently, an Arabidopsis thaliana proteome database was released examining gene expression and [protein] across organs [144] and development [145], but no such resource has been developed for abiotic stress response, making comparing this work across plant species difficult. It is also possible post-translational modifications play an important role in NCED3 activity, but none are well described at this time [146]. To the best of the author's knowledge, no other study examining transcript, protein, and metabolite concentrations has been performed that includes NCED3 and ABA at this time.

\section{Other factors and regulatory mechanisms may impact ABA metabolism}

Three levels of ABA regulation were examined in this study. However, numerous other steps of regulation may impact [ABA], [ABA metabolite], and the physiological responses a plant has to WD. For these reasons, the response of a plant to a short, long, moderate, or severe WD may be vastly different. Many levels of the biochemical regulation of WD response and ABA biosynthesis require characterization. NCED3 has uncharacterized phosphorylation and other potential post-translational modification sites that may affect activity or localization [147]. NCED3 may also interact with other proteins with unknown consequences. Alternative splicing and alternative 3'UTR usage may affect transcript function, lifetime, and localization may potentially significantly impact or optimize a WD response based on the severity and duration of the stress [12, 148, 149]. Signal peptides like CLE25 [109], miRNA [14], siRNA [15], and other regulatory molecules likely play important roles in ABA metabolism and WD response and require further investigation. 
Lastly, grafting interactions may affect ABA metabolism, signaling, sensitivity, and physiological responses to WD. Traditionally, grapevines are cultivated by grafting a desirable fruit bearing scion onto an adventitious rootstock selected based on environmental conditions. Grafting enables desirable traits of a rootstock like disease resistance or salt tolerance to be conferred to the scion [150]. Previously, grafting was not found to affect the physiological response of grapevine to a short WD [151]. However, grafting has indisputable effects on both the rootstock and scion [14, 117, 152, 153], and the molecular effects grafting may have on ABA metabolism and WD response remain unresolved. Further experiments are underway to better understand the complex effect grafting has on ABA metabolism and WD response in grapevine under long-term and cyclic WD.

\section{Conclusions}

ABA biochemistry was investigated at three levels of regulation (transcript, protein, and metabolite) across three experiments of varying severity and duration in the leaves and roots of four grapevine species. RNA-Seq analysis and metabolite quantification demonstrated ABA metabolism was a major WD response. ABA metabolites could in part be explained by upstream ABA metabolism gene transcript abundances. Gene expression profiling, DEA, WGCNA, GO enrichment analysis, and RT-qPCR supported NCED3 as a key WD response gene. NCED3 was highly expressed and significantly differentially expressed in response to WD in leaves and roots of all four species. NCED3 was also the only ABA metabolism gene identified as a hub gene in WGCNA modules corresponding both to WD and the ABA metabolites. Finally, NCED3 was supported as a hub gene in GO by the presence of biosynthetic and abiotic stress response GO terms in the WD and ABA metabolite correlated modules. Western blots demonstrated NCED3 protein abundance did not appear to change in response to WD indicating that protein activity may be different from protein abundance. There was unique and specific ABA metabolism regulation and WD response that occurred in the leaves and roots. Grapevine species demonstrated a spectrum of physiological, biochemical, and metabolic responses to different WD conditions. These responses depended on the duration and severity of WD. RA responded earlier to long-term WD with higher NCED3 transcripts and [ABA] while maintaining physiological activity longer at the cost of water availability. During long-term WD, RI generally experienced a smaller spike in NCED3 transcripts and [ABA], which were maintained at a constant level throughout the stress in parallel with quick closing stomata. NCED3 transcript abundance did not mirror [ABA] in all species during short-term WD, indicating the species have distinct ABA metabolism responses to different WD severities and durations. Thus, this study shows that ABA metabolism and regulation in grapevine species is variable and complex. Additional studies are needed to further elucidate this interesting and important topic in order to produce better more drought tolerant crops.

\section{Materials And Methods}

\section{Plant material and growth conditions}


Vitis vinifera (L.) cv. Cabernet Sauvignon clone 8 (CS) obtained from Inland Desert Nursery (Benton City, Washington, USA), Vitis champinii (RA), Vitis riparia (RI), and a Vitis vinifera x girdiana hybrid (SC2) obtained from the Plant Foundation Services at UC Davis (Davis, CA USA) were grown in a greenhouse. Greenhouse conditions ranged from $21-26^{\circ} \mathrm{C}, 20-50 \%$ relative humidity, and had average mid-day light intensities of $1200 \mu$ moles $\mathrm{m}^{-2} \mathrm{~s}^{-1}$. Supplemental light was applied using $1000 \mathrm{~W}$ high pressure sodium lamps to maintain a $16 \mathrm{~h}$ light and $8 \mathrm{~h}$ dark cycle. Pots were elevated $7.5 \mathrm{~cm}$ off the floor on perforated black plastic flats in a random design for species, treatment, and harvest date. Cuttings from mother plants were hydroponically propagated in aerated pH 5.5 water trays. When roots were established, cuttings were transferred to Stuewe and Son's Anderson AB39 pots $(0.96 \mathrm{~L})$ consisting of $\sim 1.0 \mathrm{~kg}$ Quikrete medium grain sand and $80 \mathrm{~g}$ of fritted clay for plants used for RNA-Seq and small pot experiments. Propagates were allowed to acclimate to potting conditions for one month before beginning experiments. Plants were irrigated with Cramer's complete nutrient solution $\left(1.5 \mathrm{mM} \mathrm{Ca}\left(\mathrm{NO}_{3}\right)_{2}, 2 \mathrm{mM}\right.$ $\mathrm{KNO}_{3}, 0.6 \mathrm{mM} \mathrm{MgSO}_{4}, 1 \mathrm{mM} \mathrm{KH}_{2} \mathrm{PO}_{4}, 1.5 \mathrm{mM} \mathrm{CaCl}_{2}, 36 \mu \mathrm{M} \mathrm{Fe}_{2}{ }^{+}$Sprint 330, $1 \mu \mathrm{M} \mathrm{MnSO}_{4}, 0.5 \mu \mathrm{M} \mathrm{CuSO}_{4}$, $20 \mu \mathrm{MnSO}_{4}, 20 \mu \mathrm{M} \mathrm{H}_{3} \mathrm{BO}_{3}$, and $\left.0.01 \mu \mathrm{M}\left(\mathrm{NH}_{4}\right)_{6} \mathrm{Mo}_{7} \mathrm{O}_{24}\right)$.

\section{Small pot experiments}

After pots were prepared, $100 \%$ relative soil water content (RSWC) was measured as the weight of the individual pot two hours after irrigation to the point of saturation (water flowing from bottom of pot). Each pot was covered with aluminum foil to minimize evaporation. Experiments performed in the small pots included 1) water deficit and control treatments for one-and two week moderate WD used for RNASeq, ABA metabolite derivative quantification, and physiological measurements 2) one-week severe WD and Control treatments to support the original RNA-Seq experiment and quantify NCED3 transcripts and NCED3 protein as well as ABA (in addition to original measurements reported elsewhere [8]).

For the original moderate WD RNA-Seq experiment previously described [8], 3-5 individual CS, RA, RI, and SC vines were subjected to one or two week of moderate water deficit, or well-watered daily complete nutrient solution. At W1 and W2 of treatment various measurements were taken [8]. Total plant leaves (excluding petiole) and roots were harvested after W1 and W2 of treatment. Sand was removed from root samples by briefly washing in room temperature tap water for 10 seconds and patting dry on paper towels for an additional 10 seconds. Samples were immediately frozen in liquid nitrogen and stored at $-80^{\circ} \mathrm{C}$.

For the one-week severe WD experiment, 3-5 individual CS, RA, RI, and SC vines were subjected to W1 of severe water deficit or watered daily to saturation with a complete nutrient solution. At W1 of treatment various measurements were taken [8], and total plant leaves (excluding petiole) and roots were harvested. Sand was removed from root as in the moderate WD experiment. Samples were immediately frozen in liquid nitrogen and stored at $-80^{\circ} \mathrm{C}$.

\section{Rapid dehydration experiments}


The day before the experiment, the first mature leaf (5-6th node from the apical meristem) of CS, RA, and RI vines were measured from petiole attachment point to the tip of the leaf down the midvein and marked with a tag to ensure leaves of similar developmental stage and leaf area were used for this experiment. Also, on the day before the experiment, dehydration chambers were prepared. Air tight dehydration chambers (1.2 L Rubbermaid Takealong ( ) container, Newell Rubbermaid, Atlanta, GA, USA) were prepared as before [135], containing $50 \mathrm{~mL} 333 \mathrm{mM} \mathrm{NaCl}$ or DI water (for rapid dehydration and Control treatment, respectively) and a wire support grid. Dehydration chambers were placed in a $27^{\circ} \mathrm{C}$ growth chamber $\left(\sim 200 \mu \mathrm{mol} \mathrm{m} \mathrm{m}^{-2} \mathrm{~s}^{-1}\right)$ to equilibrate overnight. The following day, two hours before solar noon, the marked leaves were cut under water, weighed, and quickly placed in a dehydration chamber $(\sim 30$ secs). WD leaves were placed abaxial side up with no contact to the salt solution. Control leaves were placed adaxial side up with petioles dipping into the water to prevent dehydration and provide petiole water-feeding. Five leaves were prepared per species per treatment per round. Leaves were collected after $2,4,8$, and 24 hours of treatment. At these times three leaves were combined and immediately frozen in liquid nitrogen. These leaves were later used for NCED3 transcript, NCED3 protein, and ABA quantification. Photosynthesis and stomatal conductance were measured on a fourth leaf using a portable photosynthesis system (LICOR model 6400XT, Lincoln, NE, USA) set at $400 \mu \mathrm{mol} \mathrm{s}^{-1}$ flow rate, $400 \mu \mathrm{mol} \mathrm{mol}{ }^{-1}$ reference $\mathrm{CO}_{2}$, with a leaf temperature at $27^{\circ} \mathrm{C}$ and PAR $1000 \mu \mathrm{mol} \mathrm{m}^{-2} \mathrm{~s}^{-1}$. After LICOR measurements, leaf water potential was measured with a Plant Water Status Console (Soilmoisture Equipment Corp., CA, USA) on the same fourth leaf. The fourth leaf was placed in a syringe and frozen in liquid nitrogen. Later, the syringe was thawed and $10 \mu \mathrm{L}$ was extracted to measure osmotic potential in triplicate. Osmotic potential was measured with a vapor pressure osmometer (VaPro 5600 EliTechGroup). Turgor was calculated as the difference between leaf water potential and osmotic pressure [102]. A fifth leaf was used to measure leaf water content. Water content was calculated as the difference between the leaf fresh weight at harvest and the leaf dry weight divided by the difference between turgid leaf weight and the leaf dry weight [154]. All leaves were weighed before final measurements were performed to determine water loss. This experiment was performed five times with each leaf coming from an individual vine. Three leaves were combined for NCED3 transcripts, NCED3 protein, and ABA quantification per round to have enough material for all measurements.

\section{RNA-Seq analysis}

Reads were trimmed as previously described using trimmomatic v0.36 [155] and sample quality was confirmed with FastQC v0.11.5 [156]. Since the original analysis of the RNA-Seq data series PRJNA516950 with the PN40024 V2 assembly V1 annotation [157], novel Vitis genomes including CS [98], RI [158], Chardonnay [159], Zinfandel [160], Carménère [161], and many others [162] were released. To improve the original analysis, PRJNA516950 was analyzed with the CS clone 8 v1.0 genome due to the quality, accessibility, and overlap with the experimental design. After filtering, reads were aligned against the CS genome [98] with HISAT2 v2.0.5 [163]. Counts were obtained with featureCounts v1.5.1 [164]. Differentially expressed genes with an adjusted $p$-value $\leq 0.05$ were identified with DESeq2 v1.26.0 [165]. Contrasts of interest included comparing each species $x$ week $x$ organ Control and WD as well as comparing WD RA $x$ organ $x$ week to those of CS, RI, and SC. All heatmaps were drawn with 
ComplexHeatmap v2.2.0 [166], dendextend v1.13.4 [167], and circlize v0.4.8 [168] to extract contrasts of interest in RStudio. WGCNA version 1.41 [100] were performed per organ as previously described [8]. Topological Overlap Matrix (TOM) was used to detect modules using the DynamicTreecut algorithm with a minimal module size of 30 and a branch merge cut height of 0.25 . The module eigengenes were used to evaluate the association among the modules for species, treatments, weeks, and various ABA metabolites. GO enrichment analysis was performed with TopGO v2.38.1 [169] using biological process terms corresponding to the CS clone 8 v1.0 genome.

\section{ABA quantification}

ABA and ABA related metabolites were quantified with HPLC-MS/MS using a methanol-based extraction as previously described [96]. Briefly, ABA derivative quantification from RNA-Seq, severe W1 WD, and rapid dehydration samples was performed as previously described with a methanol, formic acid, water (15:1:4) extraction and deuterated standards. Samples were purified with Oasis ${ }^{\circledR}$ HLB $3.5 \mathrm{ml}$ columns (Waters ${ }^{\circledR}$ ) and eluted with methanol. Samples were reconstituted in acetonitrile, water, and formic acid (15:85:0.1) and separated with an Aligent ${ }^{\mathrm{TM}}$ Zorbax $^{\circledR}$ Extend $\mathrm{C}_{18}$ column $(201 \times 150 \mathrm{~mm} ; 5 \mu \mathrm{m})$ with Opti-Solve ${ }^{\circledR}$ 2micron guard column (Optimize Technologies ${ }^{\mathrm{TM}}$, USA). A binary gradient of LC-MS grade acetonitrile (Thermofisher Scientific) acidified with $0.1 \%$ formic acid and HPLC grade water (Thermofisher Scientific) also acidified with $0.1 \%$ formic acid were used to resolve ABA derivatives. Mass spectrometry was carried out using multiple reaction monitoring (MRM) using a hybrid triple quadrupole/linear ion trap 4000 QTRAP LC-MS/MS instrument equipped with a Turbo V source (Applied Biosystems ${ }^{\text {TM }}$, USA) with a 0.2 $\mathrm{mL} / \mathrm{min}$ flow rate under the conditions previously described. D6 deuterated ABA (Toronto Research chemicals) was used as an internal standard for all samples and D4 deuterated ABA (OIChemIm) was used as an extraction efficiency standard for one- and two-week moderate and one-week severe WD samples only.

\section{RT-qPCR}

RT-qPCR was performed as previously described [8]. Briefly, all samples were ground with a mortar and pestle under liquid nitrogen. RNA extraction was performed with a previously described CTAB based extraction and $\mathrm{LiCl}$ precipitation following cleanup with the Spectrum ${ }^{\text {TM }}$ Plant Total RNA kit (SigmaAldrich). All RNA extractions were treated with RNAse-free DNase I (Qiagen) to remove genomic DNA contamination. Samples were quantified on a nanodrop, and RNA quality was confirmed with gel electrophoresis by loading 250ng RNA on a $1.2 \%$ gel as well as checked for the presence of gDNA with a GoTaq Green-LAR based PCR analyzed with electrophoresis on a $2 \%$ gel employing 400ng of RNA. Primers were designed using NCBI Primer-BLAST. Primer sequences were previously described [8]. Reference genes ( $A C T 7$ and GAPDH) were chosen for consistent band intensity under the various experimental conditions determined with Go-Taq Green based PCR reaction. The PCR reaction included $95^{\circ} \mathrm{C}$ for $2 \mathrm{~min}, 35$ cycles of $95^{\circ} \mathrm{C}$ for $30 \mathrm{~s}, 62^{\circ} \mathrm{C}$ for $25 \mathrm{~s}$ and $72{ }^{\circ} \mathrm{C}$ for $25 \mathrm{~s}$. Primer efficiencies were verified on purified PCR products (Machery-Nagel NucleoSpin ${ }^{\circledR}$ Gel and PCR Clean-up kit) and were considered at $100 \%$ for the gene expression calculations. All reactions were performed on a Bio-Rad RealTime thermal cycler CFX96 with the following protocol: $95^{\circ} \mathrm{C}$ for 3 mins; 40 cycles of $95^{\circ} \mathrm{C}$ for $10 \mathrm{~s}, 60^{\circ} \mathrm{C}$ 
for $15 \mathrm{~s}$. Fluorescence was recorded after each cycle and melting curve analysis was performed from $65^{\circ} \mathrm{C}$ to $95^{\circ} \mathrm{C}$. Reference genes were selected based on a low coefficient of variation of expression reported in literature and uniform expression for all cDNA samples for each of the above-described experiments. NCED3 transcript abundance for the W1 severe WD experiment was previously reported [8].

\section{Western Blots}

The IRC was spiked with $2 \mathrm{kDa}$ peptides the antibodies were designed against (Additional File 10) to act as a positive control. Several postulated NCED3 degradation products or subunits were also detected (Additional File 10), but only the $67 \mathrm{kDa}$ band was used for relative quantification.

Relative NCED3 protein abundance was quantified using Western Blots with internal NCED3 peptide standards and StainFree membrane normalization using an IRC loaded on each gel [101]. Proteins were extracted as previously described with chloroform/methanol [170] for one-week severe WD samples and in $2 x$ laemmli buffer for rapid dehydration samples. Total protein extracts were quantified with the Pierce BCA Protein Assay Kit (Thermo Scientific) according to the manufacturer's instructions. Total protein extract $(35 \mu \mathrm{g})$ was loaded on 12.5\% TGX stain free polyacrylamide gels (BioRad) with $5 \mu \mathrm{L}$ of precision plus all blue standard ladder (BioRad) and a CS Control IRC (35 $\mu \mathrm{g})$ spiked with $15.75 \mathrm{ng}$ of NCED3 peptide standard (Pacific Immunology) that acted as a positive control. Proteins were transferred onto a PVDF membrane (BioRad) using a Trans-Blot Turbo (BioRad) and imaged with a Chemidoc (BioRad) for total protein [101]. Membranes were blocked and probed with NCED3 (rabbit) primary antibodies (each 1:1000) (a 1:1 mix of antiNCED3.1 and 3.2 (Pacific Immunology)) (Additional File 9) followed by goat anti-rabbit-HRP (BioRad) secondary antibody (1:000). ECL-Spray (Advansta) was applied uniformly to the membrane, and the membranes were imaged with a Chemidoc (BioRad) for 12 secs. NCED3 protein in samples was quantified in ImageLab (BioRad) relative to the IRC ran on each gel and normalized to a fragment of total protein free of transfer artifacts from a StainFree membrane image [101]. Antibody (antiNCED3.1, antiNCED3.2, and antiNCED3.3) target sequences were determined to be NCED3 specific relative to the other CS NCEDs via BLAST, unique from each other, and present in available Vitis genomes (PN40024 [99], CS [171], and RI [158]) (Additional File 9). Antibody detection was linearly related to the amount of protein loaded and deemed to be sufficient for relative quantification of protein amount (Additional File 9).

\section{Statistical analysis}

Statistical analysis was performed comparing multiple means including one-, two-, three-, and four-way ANOVAs after assumptions were met. Non-normal data was box-cox transformed to meet normality and homoscedastic assumptions [172]. Post Hoc tests were performed with Tukey's Test HSD for comparisons between species, treatments, and time points after assumptions were met. Asterisks indicate statistical significance from ANOVA $(*=p \leq 0.05, * *=p \leq 0.01, * * *=p \leq 0.001)$. Letters indicate statistical significance between the multiple comparisons. The error rate $a=0.05$ was used in all comparisons. Statistical analyses and all other R-based analyses were performed using $\mathrm{R}$ version 3.6.3in RStudio version 1.2.13335 [173]. 


\section{Abbreviations}

$\Psi_{\text {stem }}=$ Stem water potential

$\Psi_{\mathrm{S}}=$ Solute pressure

$\Psi_{\mathrm{p}}=$ turgor pressure

7’OH ABA = 7' hydroxy Abscisic acid

BETA-OHASE = ß-CAROTENE HYDROXYLASE

$A A O=A B A$ ALDEHYDE OXIDASE

ABA1 = ZEAXANTHIN EPOXIDASE

ABA2 = XANTHOXIN DEHYDROGENASE

ABA3 = MOLYBDENUM COFACTOR SULFURASE

$\mathrm{ABA}=\mathrm{Abscisic}$ acid

ABA-GE = Abscisic acid glucose ester

$\mathrm{ABF}=\mathrm{ABSCISIC}$ ACID RESPONSIVE ELEMENT-BINDING FACTOR

$A B I=A B A$ INSENSITIVE

AREB $=$ ABA-RESPONSIVE ELEMENT BINDING PROTEIN

$B G=ß-d-G L U C O S I D A S E$

$B G L U=ß-d-G L U C O S I D A S E$

BLAST = Basic local alignment search tool

$\mathrm{bp}=$ Base pair

$\mathrm{CS}=$ Vitis vinifera (L.) cv. Cabernet Sauvignon clone 8.1

DEA = Differential expression analysis

DI = De-ionized

DPA $=$ Dihydrophaseic acid

DW = Dry weight 
GGPP = Geranylgeranyl-diphosphate

Gs $=$ Stomatal conductance

HSD = Honest Significant Difference

$\mathrm{kDa}=$ kilodalton

$L A R=$ Leucoanthocyanidin reductase

MEP = methylerythritol phosphate

$\min =$ Minute

NCED = nine-cis-epoxycarotenoid dioxygenase

NeoPA $=$ Neophaseic acid

$\mathrm{NRQ}=$ Normalized relative quantity

NRT1.2 = NITRATE TRANSPORTER 1.2

NXS = NEOXANTHIN SYNTHASE

$\mathrm{PA}=$ Phaseic acid

PP2C = PROTEIN PHOSPHATASE 2C

Ps = Photosynthesis

PYL = PYRABACTIN RESISTANCE-LIKE

PYR = PYRABACTIN RESISTANCE

$\mathrm{RA}=$ Vitis champinii (Ramsey)

RCAR = Regulatory components of ABA receptors

RD $=$ RESPONSIVE TO DESSICATION

RSWC $=$ Relative soil water content

$\mathrm{RI}=$ Vitis Riparia (Riparia Gloire)

RNA-Seq = Ribonucleic acid-sequencing

RT-qPCR = Real time-quantitative polymerase chain reaction 
$s=$ Second

$\mathrm{SA}=$ Surface area

$\mathrm{SE}=$ Standard error of the mean

SNRK2 = SNF1-RELATED PROTEIN KINASE 2

STD = Standard deviation

$\mathrm{SC} 2=$ Vitis vinifera $\times$ Vitis girdiana $(\mathrm{SC} 2)$

TFs $=$ Transcription factors

UGT = UDP-GLUCOSE TRANSFERASE

Vs. $=$ Versus

$W=$ Watt

W1 $=$ Week one

W2 $=$ Week two

WD $=$ Water deficit

WGNCA $=$ Weighted gene network co-expression analysis

ZEP = ZEAXANTHIN EPOXIDASE

\section{Declarations}

\section{Ethics approval and consent to participate}

Not applicable.

\section{Consent for publication}

Not applicable.

\section{Availability of data and material}

RNA-Seq data were deposited in the Sequence Read Archive (SRA) database with the accession number PRJNA516950 in a previous work [8]. All other data available upon request to corresponding author. 


\section{Competing interests}

The authors declare they have no competing interests. Grant R. Cramer was a member of the editorial board (section editor) of this journal previously.

\section{Funding}

This research was supported by the USDA National Institute of Food and Agriculture Hatch project 1015139 and the National Institute of General Medical Sciences (GM103440) from the National Institutes of Health.

\section{Authors' contributions}

GC obtained funding for this work. HT and NC performed small pot experiments. HT designed and performed rapid dehydration experiments. NC and HT performed RNA-Seq analysis and WGCNA. HT performed $\mathrm{GO}$ enrichment analysis. LD performed ABA metabolite quantification for the one and twoweek moderate WD experiment. HT performed ABA extraction and quantification under the guidance of LD for the one-week severe WD and rapid dehydration experiments. HT performed RT-qPCR. HT and KG optimized Western Blot conditions. HT and KG extracted proteins. HT performed Western Blots, and quantified relative NCED3 abundance. HT managed and analyzed all data and wrote the body of the paper. All authors reviewed, edited, and approved the final manuscript.

\section{Author Details}

${ }^{1}$ Department of Biochemistry and Molecular Biology, University of Nevada, Reno, Reno, NV 89557, USA; 2Present address: Department of Viticulture and Enology, University California Davis, Davis California, USA

\section{Acknowledgements}

The authors thank all the undergraduate and Biotechnology student members of the laboratory for their technical assistance.

\section{References}

1. Um M-J, Kim Y, Park D, Kim J. Effects of different reference periods on drought index (SPEI) estimations from 1901 to 2014. Hydrol Earth Syst Sci. 2017;21:4989-5007.

2. Monteverde C, De Sales F. Impacts of global warming on southern California's winegrape climate suitability. Adv Clim Change Res. 2020;11:279-93. 
3. Cabré F, Nuñez M. Impacts of climate change on viticulture in Argentina. Reg Environ Change. 2020;20:12.

4. Dai A. Increasing drought under global warming in observations and models. Nat Clim Change. 2013;3:52-8.

5. Lesk C, Rowhani P, Ramankutty N. Influence of extreme weather disasters on global crop production. Nature. 2016;529:84-7.

6. Asensi-Fabado M-A, Amtmann A, Perrella G. Plant responses to abiotic stress: The chromatin context of transcriptional regulation. Biochim Biophys Acta BBA - Gene Regul Mech. 2017;1860:106-22.

7. Kim J-M, To TK, Ishida J, Morosawa T, Kawashima M, Matsui A, et al. Alterations of Lysine Modifications on the Histone H3 N-Tail under Drought Stress Conditions in Arabidopsis thaliana. Plant Cell Physiol. 2008;49:1580-8.

8. Cochetel N, Ghan R, Toups HS, Degu A, Tillett RL, Schlauch KA, et al. Drought tolerance of the grapevine, Vitis champinii cv. Ramsey, is associated with higher photosynthesis and greater transcriptomic responsiveness of abscisic acid biosynthesis and signaling. BMC Plant Biol. 2020;20. doi:10.1186/s12870-019-2012-7.

9. Chong GL, Foo MH, Lin W-D, Wong MM, Verslues PE. Highly ABA-Induced 1 (HAl1)-Interacting protein HIN1 and drought acclimation-enhanced splicing efficiency at intron retention sites. Proc Natl Acad Sci. 2019;116:22376-85.

10. Lei L, Shi J, Chen J, Zhang M, Sun S, Xie S, et al. Ribosome profiling reveals dynamic translational landscape in maize seedlings under drought stress. Plant J. 2015;84:1206-18.

11. Srivastava AK, Lu Y, Zinta G, Lang Z, Zhu J-K. UTR dependent control of gene expression in plants. Trends Plant Sci. 2018;23:248-59.

12. Chakrabarti M, Dinkins RD, Hunt AG. Genome-wide atlas of alternative polyadenylation in the forage legume red clover. Sci Rep. 2018;8:11379.

13. Bouzroud S, Gouiaa S, Hu N, Bernadac A, Mila I, Bendaou N, et al. Auxin Response Factors (ARFs) are potential mediators of auxin action in tomato response to biotic and abiotic stress (Solanum lycopersicum). PLoS ONE. 2018;13. doi:10.1371/journal.pone.0193517.

14. Pagliarani $C$, Vitali M, Ferrero $M$, Vitulo N, Incarbone $M$, Lovisolo $C$, et al. The Accumulation of miRNAs Differentially Modulated by Drought Stress Is Affected by Grafting in Grapevine1[OPEN]. Plant Physiol. 2017;173:2180-95.

15. Jung I, Ahn H, Shin S-J, Kim J, Kwon H-B, Jung W, et al. Clustering and evolutionary analysis of small RNAs identify regulatory siRNA clusters induced under drought stress in rice. BMC Syst Biol. 2016;10:115.

16. Ghimire S, Tang X, Zhang N, Liu W, Si H. SUMO and SUMOylation in plant abiotic stress. Plant Growth Regul. 2020. doi:10.1007/s10725-020-00624-1.

17. Hashiguchi A, Komatsu S. Impact of Post-Translational Modifications of Crop Proteins under Abiotic Stress. Proteomes. 2016;4. doi:10.3390/proteomes4040042. 
18. Guerra D, Crosatti C, Khoshro HH, Mastrangelo AM, Mica E, Mazzucotelli E. Post-transcriptional and post-translational regulations of drought and heat response in plants: a spider's web of mechanisms. Front Plant Sci. 2015;6. doi:10.3389/fpls.2015.00057.

19. Kobayashi Y, Murata M, Minami H, Yamamoto S, Kagaya Y, Hobo T, et al. Abscisic acid-activated SNRK2 protein kinases function in the gene-regulation pathway of ABA signal transduction by phosphorylating ABA response element-binding factors. Plant J. 2005;44:939-49.

20. Liu X, Yue Y, Li B, Nie Y, Li W, Wu W-H, et al. A G Protein-Coupled Receptor Is a Plasma Membrane Receptor for the Plant Hormone Abscisic Acid. Science. 2007;315:1712-6.

21. Pandey S, Nelson DC, Assmann SM. Two Novel GPCR-Type G Proteins Are Abscisic Acid Receptors in Arabidopsis. Cell. 2009;136:136-48.

22. Shen Y-Y, Wang X-F, Wu F-Q, Du S-Y, Cao Z, Shang Y, et al. The Mg-chelatase H subunit is an abscisic acid receptor. Nature. 2006;443:823-6.

23. Park S-Y, Fung P, Nishimura N, Jensen DR, Fujii H, Zhao Y, et al. Abscisic Acid Inhibits Type 2C Protein Phosphatases via the PYR/PYL Family of START Proteins. Science. 2009;:1173041.

24. Nakashima K, Fujita Y, Kanamori N, Katagiri T, Umezawa T, Kidokoro S, et al. Three Arabidopsis SnRK2 Protein Kinases, SRK2D/SnRK2.2, SRK2E/SnRK2.6/OST1 and SRK2I/SnRK2.3, Involved in ABA Signaling are Essential for the Control of Seed Development and Dormancy. Plant Cell Physiol. 2009;50:1345-63.

25. Mustilli A-C, Merlot S, Vavasseur A, Fenzi F, Giraudat J. Arabidopsis OST1 Protein Kinase Mediates the Regulation of Stomatal Aperture by Abscisic Acid and Acts Upstream of Reactive Oxygen Species Production. Plant Cell. 2002;14:3089-99.

26. Furihata T, Maruyama K, Fujita Y, Umezawa T, Yoshida R, Shinozaki K, et al. Abscisic acid-dependent multisite phosphorylation regulates the activity of a transcription activator AREB1. Proc Natl Acad Sci U S A. 2006;103:1988-93.

27. Yoshida T, Fujita Y, Sayama H, Kidokoro S, Maruyama K, Mizoi J, et al. AREB1, AREB2, and ABF3 are master transcription factors that cooperatively regulate ABRE-dependent ABA signaling involved in drought stress tolerance and require ABA for full activation. Plant J. 2010;61:672-85.

28. Umezawa T, Sugiyama N, Takahashi F, Anderson JC, Ishihama Y, Peck SC, et al. Genetics and Phosphoproteomics Reveal a Protein Phosphorylation Network in the Abscisic Acid Signaling Pathway in Arabidopsis thaliana. Sci Signal. 2013;6:rs8-rs8.

29. Vlad F, Turk BE, Peynot P, Leung J, Merlot S. A versatile strategy to define the phosphorylation preferences of plant protein kinases and screen for putative substrates. Plant J. 2008;55:104-17.

30. Wang P, Xue L, Batelli G, Lee S, Hou Y-J, Van Oosten MJ, et al. Quantitative phosphoproteomics identifies SnRK2 protein kinase substrates and reveals the effectors of abscisic acid action. Proc Natl Acad Sci U S A. 2013;110:11205-10.

31. Finkelstein RR, Lynch TJ. The Arabidopsis Abscisic Acid Response Gene ABI5 Encodes a Basic Leucine Zipper Transcription Factor. Plant Cell. 2000;12:599-609. 
32. Finkelstein R, Gampala SSL, Lynch TJ, Thomas TL, Rock CD. Redundant and Distinct Functions of the ABA Response Loci ABA-INSENSITIVE(ABI)5 and ABRE-BINDING FACTOR (ABF)3. Plant Mol Biol. 2005;59:253-67.

33. Parent B, Hachez C, Redondo E, Simonneau T, Chaumont F, Tardieu F. Drought and Abscisic Acid Effects on Aquaporin Content Translate into Changes in Hydraulic Conductivity and Leaf Growth Rate: A Trans-Scale Approach. Plant Physiol. 2009;149:2000-12.

34. Zarrouk O, Garcia-Tejero I, Pinto C, Genebra T, Sabir F, Prista C, et al. Aquaporins isoforms in cv. Touriga Nacional grapevine under water stress and recovery-Regulation of expression in leaves and roots. Agric Water Manag. 2016;164:167-75.

35. Wong DCJ, Zhang L, Merlin I, Castellarin SD, Gambetta GA. Structure and transcriptional regulation of the major intrinsic protein gene family in grapevine. BMC Genomics. 2018;19:248.

36. Cottle W, Kolattukudy PE. Abscisic Acid Stimulation of Suberization. Plant Physiol. 1982;70:775-80.

37. Lippold F, Sanchez DH, Musialak M, Schlereth A, Scheible W-R, Hincha DK, et al. AtMyb41 Regulates Transcriptional and Metabolic Responses to Osmotic Stress in Arabidopsis. Plant Physiol. 2009;149:1761-72.

38. Li T, Zhang Y, Liu Y, Li X, Hao G, Han Q, et al. Raffinose synthase enhances drought tolerance through raffinose synthesis or galactinol hydrolysis in maize and Arabidopsis plants. J Biol Chem. 2020;295:8064-77.

39. Taji T, Ohsumi C, luchi S, Seki M, Kasuga M, Kobayashi M, et al. Important roles of drought- and coldinducible genes for galactinol synthase in stress tolerance in Arabidopsis thaliana. Plant J. 2002;29:417-26.

40. Abe H, Urao T, Ito T, Seki M, Shinozaki K, Yamaguchi-Shinozaki K. Arabidopsis AtMYC2 (bHLH) and AtMYB2 (MYB) Function as Transcriptional Activators in Abscisic Acid Signaling. Plant Cell. 2003;15:63-78.

41. Tu M, Wang X, Zhu Y, Wang D, Zhang X, Cui Y, et al. VIbZIP30 of grapevine functions in drought tolerance via the abscisic acid core signaling pathway. preprint. Plant Biology; 2017. doi: $10.1101 / 231811$.

42. Frébort I, Kowalska M, Hluska T, Frébortová J, Galuszka P. Evolution of cytokinin biosynthesis and degradation. J Exp Bot. 2011;62:2431-52.

43. Kasahara H, Hanada A, Kuzuyama T, Takagi M, Kamiya Y, Yamaguchi S. Contribution of the mevalonate and methylerythritol phosphate pathways to the biosynthesis of gibberellins in Arabidopsis. J Biol Chem. 2002;277:45188-94.

44. Fujioka S, Yokota T. Biosynthesis and Metabolism of Brassinosteroids. Annu Rev Plant Biol. 2003;54:137-64.

45. Alder A, Jamil M, Marzorati M, Bruno M, Vermathen M, Bigler P, et al. The Path from -Carotene to Carlactone, a Strigolactone-Like Plant Hormone. Science. 2012;335:1348-51.

46. Rodríguez-Concepción M, Boronat A. Elucidation of the Methylerythritol Phosphate Pathway for Isoprenoid Biosynthesis in Bacteria and Plastids. A Metabolic Milestone Achieved through Genomics. 
Plant Physiol. 2002;130:1079-89.

47. Welsch R, Beyer P, Hugueney P, Kleinig H, von Lintig J. Regulation and activation of phytoene synthase, a key enzyme in carotenoid biosynthesis, during photomorphogenesis. Planta. 2000;211:846-54.

48. Welsch R, Wüst F, Bär C, Al-Babili S, Beyer P. A Third Phytoene Synthase Is Devoted to Abiotic StressInduced Abscisic Acid Formation in Rice and Defines Functional Diversification of Phytoene Synthase Genes. Plant Physiol. 2008;147:367-80.

49. Bartley GE, Scolnik PA, Beyer P. Two Arabidopsis thaliana carotene desaturases, phytoene desaturase and zeta-carotene desaturase, expressed in Escherichia coli, catalyze a poly-cis pathway to yield prolycopene. Eur J Biochem. 1999;259:396-403.

50. Linden H, Misawa N, Saito T, Sandmann G. A novel carotenoid biosynthesis gene coding for zetacarotene desaturase: functional expression, sequence and phylogenetic origin. Plant Mol Biol. 1994;24:369-79.

51. Ruiz-Sola MÁ, Rodríguez-Concepción M. Carotenoid Biosynthesis in Arabidopsis: A Colorful Pathway. Arab Book Am Soc Plant Biol. 2012;10. doi:10.1199/tab.0158.

52. Zeng J, Wang C, Chen X, Zang M, Yuan C, Wang X, et al. The lycopene $\beta$-cyclase plays a significant role in provitamin A biosynthesis in wheat endosperm. BMC Plant Biol. 2015;15. doi:10.1186/s12870-015-0514-5.

53. The zeaxanthin biosynthesis enzyme ${ }^{2}$-carotene hydroxylase is involved in myxoxanthophyll synthesis in Synechocystis sp. PCC 6803. FEBS Lett. 1999;454:247-51.

54. Du H, Wang N, Cui F, Li X, Xiao J, Xiong L. Characterization of the $\beta$-Carotene Hydroxylase Gene DSM2 Conferring Drought and Oxidative Stress Resistance by Increasing Xanthophylls and Abscisic Acid Synthesis in Rice. Plant Physiol. 2010;154:1304-18.

55. Niyogi KK. PHOTOPROTECTION REVISITED: Genetic and Molecular Approaches. Annu Rev Plant Physiol Plant Mol Biol. 1999;50:333-59.

56. Hieber AD, Bugos RC, Yamamoto HY. Plant lipocalins: violaxanthin de-epoxidase and zeaxanthin epoxidase. Biochim Biophys Acta BBA - Protein Struct Mol Enzymol. 2000;1482:84-91.

57. Eskling M, Arvidsson P-O, Åkerlund H-E. The xanthophyll cycle, its regulation and components. Physiol Plant. 1997;100:806-16.

58. Qin X, Zeevaart JAD. The 9-cis-epoxycarotenoid cleavage reaction is the key regulatory step of abscisic acid biosynthesis in water-stressed bean. Proc Natl Acad Sci. 1999;96:15354-61.

59. Tan BC, Schwartz SH, Zeevaart JAD, McCarty DR. Genetic control of abscisic acid biosynthesis in maize. Proc Natl Acad Sci. 1997;94:12235-40.

60. Schwartz SH. Specific Oxidative Cleavage of Carotenoids by VP14 of Maize. Science. 1997;276:1872-4.

61. Schwartz SH, Léon-Kloosterziel KM, Koornneef M, Zeevaart JA. Biochemical characterization of the aba2 and aba3 mutants in Arabidopsis thaliana. Plant Physiol. 1997;114:161-6. 
62. González-Guzmán M, Apostolova N, Bellés JM, Barrero JM, Piqueras P, Ponce MR, et al. The ShortChain Alcohol Dehydrogenase ABA2 Catalyzes the Conversion of Xanthoxin to Abscisic Aldehyde. Plant Cell. 2002;14:1833-46.

63. Seo M, Peeters AJM, Koiwai H, Oritani T, Marion-Poll A, Zeevaart JAD, et al. The Arabidopsis aldehyde oxidase 3 (AAO3) gene product catalyzes the final step in abscisic acid biosynthesis in leaves. Proc Natl Acad Sci U S A. 2000;97:12908-13.

64. Xu Z-Y, Yoo Y-J, Hwang I. ABA Conjugates and Their Physiological Roles in Plant Cells. In: Zhang D-P, editor. Abscisic Acid: Metabolism, Transport and Signaling. Dordrecht: Springer Netherlands; 2014. p. 77-87. doi:10.1007/978-94-017-9424-4_5.

65. Todoroki Y, Narita K, Muramatsu T, Shimomura H, Ohnishi T, Mizutani M, et al. Synthesis and biological activity of amino acid conjugates of abscisic acid. Bioorg Med Chem. 2011;19:1743-50.

66. Cutler AJ, Krochko JE. Formation and breakdown of ABA. Trends Plant Sci. 1999;4:472-8.

67. Piotrowska A, Bajguz A. Conjugates of abscisic acid, brassinosteroids, ethylene, gibberellins, and jasmonates. Phytochemistry. 2011;72:2097-112.

68. Lim E-K, Doucet CJ, Hou B, Jackson RG, Abrams SR, Bowles DJ. Resolution of (+)-abscisic acid using an Arabidopsis glycosyltransferase. Tetrahedron Asymmetry. 2005;16:143-7.

69. Liu Z, Yan J-P, Li D-K, Luo Q, Yan Q, Liu Z-B, et al. UDP-Glucosyltransferase71C5, a Major Glucosyltransferase, Mediates Abscisic Acid Homeostasis in Arabidopsis1[OPEN]. Plant Physiol. 2015;167:1659-70.

70. Burla B, Pfrunder S, Nagy R, Francisco RM, Lee Y, Martinoia E. Vacuolar Transport of Abscisic Acid Glucosyl Ester Is Mediated by ATP-Binding Cassette and Proton-Antiport Mechanisms in Arabidopsis1[W][OPEN]. Plant Physiol. 2013;163:1446-58.

71. Bray EA, Zeevaart JAD. The Compartmentation of Abscisic Acid and $\beta$-d-Glucopyranosyl Abscisate in Mesophyll Cells 1. Plant Physiol. 1985;79:719-22.

72. Lehmann H, Glund K. Abscisic acid metabolism ?vacuolar/extravacuolar distribution of metabolites. Planta. 1986;168:559-62.

73. Xu Z-Y, Lee KH, Dong T, Jeong JC, Jin JB, Kanno Y, et al. A Vacuolar $\beta$-Glucosidase Homolog That Possesses Glucose-Conjugated Abscisic Acid Hydrolyzing Activity Plays an Important Role in Osmotic Stress Responses in Arabidopsis[W]. Plant Cell. 2012;24:2184-99.

74. Lee KH, Piao HL, Kim H-Y, Choi SM, Jiang F, Hartung W, et al. Activation of Glucosidase via StressInduced Polymerization Rapidly Increases Active Pools of Abscisic Acid. Cell. 2006;126:1109-20.

75. Schroeder JI, Nambara E. A Quick Release Mechanism for Abscisic Acid. Cell. 2006;126:1023-5.

76. Wasilewska A, Vlad F, Sirichandra C, Redko Y, Jammes F, Valon C, et al. An Update on Abscisic Acid Signaling in Plants and More .... Mol Plant. 2008;1:198-217.

77. Nambara E, Marion-Poll A. ABSCISIC ACID BIOSYNTHESIS AND CATABOLISM. Annu Rev Plant Biol. 2005;56:165-85. 
78. Krochko JE, Abrams GD, Loewen MK, Abrams SR, Cutler AJ. (+)-Abscisic Acid 8'-Hydroxylase Is a Cytochrome P450 Monooxygenase. Plant Physiol. 1998;118:849-60.

79. Weng J-K, Ye M, Li B, Noel JP. Co-evolution of Hormone Metabolism and Signaling Networks Expands Plant Adaptive Plasticity. Cell. 2016;166:881-93.

80. Shimomura H, Etoh H, Mizutani M, Hirai N, Todoroki Y. Effect of the minor ABA metabolite 7'-hydroxyABA on Arabidopsis ABA 8'-hydroxylase CYP707A3. Bioorg Med Chem Lett. 2007;17:4977-81.

81. Gillard DF, Walton DC. Abscisic Acid Metabolism by a Cell-free Preparation from Echinocystis lobata Liquid Endosperm. 1976;58:6.

82. Hansen H, Dörffling K. Changes of free and conjugated abscisic acid and phaseic acid in xylem sap of drought-stressed sunflower plants. J Exp Bot. 1999;50:1599-605.

83. Ikegami K, Okamoto M, Seo M, Koshiba T. Activation of abscisic acid biosynthesis in the leaves of Arabidopsis thaliana in response to water deficit. J Plant Res. 2009;122:235-43.

84. Ren H, Gao Z, Chen L, Wei K, Liu J, Fan Y, et al. Dynamic analysis of ABA accumulation in relation to the rate of ABA catabolism in maize tissues under water deficit. J Exp Bot. 2007;58:211-9.

85. Rossdeutsch L, Edwards E, Cookson SJ, Barrieu F, Gambetta GA, Delrot S, et al. ABA-mediated responses to water deficit separate grapevine genotypes by their genetic background. BMC Plant Biol. 2016;16:91.

86. Rattanakon S, Ghan R, Gambetta GA, Deluc LG, Schlauch KA, Cramer GR. Abscisic acid transcriptomic signaling varies with grapevine organ. BMC Plant Biol. 2016;16. doi:10.1186/s12870016-0763-y.

87. Guóth A, Tari I, Gallé Á, Csiszár J, Pécsváradi A, Cseuz L, et al. Comparison of the Drought Stress Responses of Tolerant and Sensitive Wheat Cultivars During Grain Filling: Changes in Flag Leaf Photosynthetic Activity, ABA Levels, and Grain Yield. J Plant Growth Regul. 2009;28:167-76.

88. Dash GK, Geda CK, Sahoo SK, Baig MJ, Swain P. Exogenously applied ABA induced changes in physio-biochemical attributes in selected rice varieties under osmotic stress. bioRxiv. 2020;:2020.05.27.116129.

89. Iqbal N, Hussain S, Raza MA, Yang C-Q, Safdar ME, Brestic M, et al. Drought Tolerance of Soybean (Glycine max L. Merr.) by Improved Photosynthetic Characteristics and an Efficient Antioxidant Enzyme Activities Under a Split-Root System. Front Physiol. 2019;10. doi:10.3389/fphys.2019.00786.

90. Iqbal N, Khan NA, Ferrante A, Trivellini A, Francini A, Khan MIR. Ethylene Role in Plant Growth, Development and Senescence: Interaction with Other Phytohormones. Front Plant Sci. 2017;8. doi:10.3389/fpls.2017.00475.

91. Perl K, Ushakov K, Pozniak Y, Yizhar-Barnea O, Bhonker Y, Shivatzki S, et al. Reduced changes in protein compared to mRNA levels across non-proliferating tissues. BMC Genomics. 2017;18:305.

92. Greenbaum D, Colangelo C, Williams K, Gerstein M. Comparing protein abundance and mRNA expression levels on a genomic scale. Genome Biol. 2003;4:117. 
93. Liu Y, Beyer A, Aebersold R. On the Dependency of Cellular Protein Levels on mRNA Abundance. Cell. 2016;165:535-50.

94. Liu Y, Aebersold R. The interdependence of transcript and protein abundance: new data-new complexities. Mol Syst Biol. 2016;12. doi:10.15252/msb.20156720.

95. Jia H, Sun W, Li M, Zhang Z. Integrated Analysis of Protein Abundance, Transcript Level, and Tissue Diversity To Reveal Developmental Regulation of Maize. J Proteome Res. 2018;17:822-33.

96. Gouthu S, Morre J, Maier CS, Deluc LG. An Analytical Method to Quantify Three Plant Hormone Families in Grape Berry Using Liquid Chromatography and Multiple Reaction Monitoring Mass Spectrometry. In: Gang DR, editor. Phytochemicals, Plant Growth, and the Environment. New York, NY: Springer New York; 2013. p. 19-36. doi:10.1007/978-1-4614-4066-6_2.

97. Sussmilch FC, Brodribb TJ, McAdam SAM. Up-regulation of NCED3 and ABA biosynthesis occur within minutes of a decrease in leaf turgor but AHK1 is not required. J Exp Bot. 2017;68:2913-8.

98. Chin C-S, Peluso P, Sedlazeck FJ, Nattestad M, Concepcion GT, Clum A, et al. Phased diploid genome assembly with single-molecule real-time sequencing. Nat Methods. 2016;13:1050-4.

99. Canaguier A, Grimplet J, Di Gaspero G, Scalabrin S, Duchêne E, Choisne N, et al. A new version of the grapevine reference genome assembly (12X.v2) and of its annotation (VCost.v3). Genomics Data. 2017;14:56-62.

100. Langfelder P, Horvath S. WGCNA: an R package for weighted correlation network analysis. BMC Bioinformatics. 2008;9:559.

101. Taylor SC, Posch A. The Design of a Quantitative Western Blot Experiment. BioMed Research International. 2014;2014:e361590. doi:https://doi.org/10.1155/2014/361590.

102. Tyree MT, Hammel HT. The Measurement of the Turgor Pressure and the Water Relations of Plants by the Pressure-bomb Technique. J Exp Bot. 1972;23:267-82.

103. Lamarque LJ, Delzon S, Toups H, Gravel A-I, Corso D, Badel E, et al. Over-accumulation of abscisic acid in transgenic tomato plants increases the risk of hydraulic failure. Plant Cell Environ. n/a n/a. doi:10.1111/pce.13703.

104. Bittner F, Oreb M, Mendel RR. ABA3 is a molybdenum cofactor sulfurase required for activation of aldehyde oxidase and xanthine dehydrogenase in Arabidopsis thaliana. J Biol Chem. 2001;276:40381-4.

105. Batool S, Uslu VV, Rajab H, Ahmad N, Waadt R, Geiger D, et al. Sulfate is Incorporated into Cysteine to Trigger ABA Production and Stomatal Closure. Plant Cell. 2018;30:2973-87.

106. Christmann A, Weiler EW, Steudle E, Grill E. A hydraulic signal in root-to-shoot signalling of water shortage: Hydraulic signalling of water shortage. Plant J. 2007;52:167-74.

107. McAdam SAM, Manzi M, Ross JJ, Brodribb TJ, Gómez-Cadenas A. Uprooting an abscisic acid paradigm: Shoots are the primary source. Plant Signal Behav. 2016;11:e1169359.

108. Carvalho DRA, Fanourakis D, Correia MJ, Monteiro JA, Araújo-Alves JPL, Vasconcelos MW, et al. Root-to-shoot $A B A$ signaling does not contribute to genotypic variation in stomatal functioning 
induced by high relative air humidity. Environ Exp Bot. 2016;123:13-21.

109. Takahashi F, Suzuki T, Osakabe Y, Betsuyaku S, Kondo Y, Dohmae N, et al. A small peptide modulates stomatal control via abscisic acid in long-distance signalling. Nature. 2018;556:235-8.

110. Assmann SM, Jegla T. Guard cell sensory systems: recent insights on stomatal responses to light, abscisic acid, and CO2. Curr Opin Plant Biol. 2016;33:157-67.

111. Duan L, Dietrich D, Ng CH, Chan PMY, Bhalerao R, Bennett MJ, et al. Endodermal ABA Signaling Promotes Lateral Root Quiescence during Salt Stress in Arabidopsis Seedlings. Plant Cell. 2013;25:324-41.

112. Chadwick AV, Burg SP. An Explanation of the Inhibition of Root Growth Caused by Indole-3-Acetic Acid. Plant Physiol. 1967;42:415-20.

113. Evans ML, Ishikawa H, Estelle MA. Responses of Arabidopsis roots to auxin studied with high temporal resolution: Comparison of wild type and auxin-response mutants. Planta. 1994;194:21522.

114. Hu J, Mitchum MG, Barnaby N, Ayele BT, Ogawa M, Nam E, et al. Potential Sites of Bioactive Gibberellin Production during Reproductive Growth in Arabidopsis. Plant Cell. 2008;20:320-36.

115. McAdam SAM, Brodribb TJ. Mesophyll Cells Are the Main Site of Abscisic Acid Biosynthesis in WaterStressed Leaves. Plant Physiol. 2018;177:911-7.

116. Zhang J, Schurr U, Davies WJ. Control of Stomatal Behaviour by Abscisic Acid which Apparently Originates in the Roots. J Exp Bot. 1987;38:1174-81.

117. Holbrook NM, Shashidhar VR, James RA, Munns R. Stomatal control in tomato with ABA-deficient roots: response of grafted plants to soil drying. J Exp Bot. 2002;53:1503-14.

118. Kuromori T, Seo M, Shinozaki K. ABA Transport and Plant Water Stress Responses. Trends Plant Sci. 2018;23:513-22.

119. Kang J, Hwang J-U, Lee M, Kim Y-Y, Assmann SM, Martinoia E, et al. PDR-type ABC transporter mediates cellular uptake of the phytohormone abscisic acid. Proc Natl Acad Sci. 2010;107:2355-60.

120. Kuromori T, Miyaji T, Yabuuchi H, Shimizu H, Sugimoto E, Kamiya A, et al. ABC transporter AtABCG25 is involved in abscisic acid transport and responses. Proc Natl Acad Sci. 2010;107:2361-6.

121. Merilo E, Jalakas P, Laanemets K, Mohammadi O, Hõrak H, Kollist $\mathrm{H}$, et al. Abscisic Acid Transport and Homeostasis in the Context of Stomatal Regulation. Mol Plant. 2015;8:1321-33.

122. Kang J, Yim S, Choi H, Kim A, Lee KP, Lopez-Molina L, et al. Abscisic acid transporters cooperate to control seed germination. Nat Commun. 2015;6:8113.

123. Léran $S$, Noguero $M$, Corratgé-Faillie $C$, Boursiac $Y$, Brachet $C$, Lacombe B. Functional Characterization of the Arabidopsis Abscisic Acid Transporters NPF4.5 and NPF4.6 in Xenopus Oocytes. Front Plant Sci. 2020;11. doi:10.3389/fpls.2020.00144.

124. Ernst L, Goodger JQD, Alvarez S, Marsh EL, Berla B, Lockhart E, et al. Sulphate as a xylem-borne chemical signal precedes the expression of ABA biosynthetic genes in maize roots. $J$ Exp Bot. 2010;61:3395-405. 
125. Goodger JQ, Schachtman DP. Re-examining the role of ABA as the primary long-distance signal produced by water-stressed roots. Plant Signal Behav. 2010;5:1298-301.

126. Winter D, Vinegar B, Nahal H, Ammar R, Wilson GV, Provart NJ. An "Electronic Fluorescent Pictograph" Browser for Exploring and Analyzing Large-Scale Biological Data Sets. PLoS ONE. 2007;2:e718.

127. Brady SM, Orlando DA, Lee J-Y, Wang JY, Koch J, Dinneny JR, et al. A High-Resolution Root Spatiotemporal Map Reveals Dominant Expression Patterns. Sci New Ser. 2007;318:801-6.

128. Kuromori T, Sugimoto E, Shinozaki K. Intertissue Signal Transfer of Abscisic Acid from Vascular Cells to Guard Cells. PLANT Physiol. 2014;164:1587-92.

129. Baier M, Gimmler H, Hartung W. The Permeability of the Guard Cell Plasma Membrane and Tonoplast. J Exp Bot. 1990;41:351-8.

130. Gambetta GA. Water Stress and Grape Physiology in the Context of Global Climate Change. J Wine Econ. 2016;11:168-80.

131. Sade N, Moshelion M. The dynamic isohydric-anisohydric behavior of plants upon fruit development: taking a risk for the next generation. Tree Physiol. 2014;34:1199-202.

132. Tardieu F, Simonneau T. Variability among species of stomatal control under fluctuating soil water status and evaporative demand: modelling isohydric and anisohydric behaviours. J Exp Bot. 1998;49 Special_Issue:419-32.

133. Schultz HR. Differences in hydraulic architecture account for near-isohydric and anisohydric behaviour of two field-grown Vitis vinifera L. cultivars during drought. Plant Cell Environ. 2003;26:1393-405.

134. Gerzon E, Biton I, Yaniv Y, Zemach H, Netzer Y, Schwartz A, et al. Grapevine Anatomy as a Possible Determinant of Isohydric or Anisohydric Behavior. Am J Enol Vitic. 2015;66:340-7.

135. Hopper DW, Ghan R, Cramer GR. A rapid dehydration leaf assay reveals stomatal response differences in grapevine genotypes. Hortic Res. 2014;1. doi:10.1038/hortres.2014.2.

136. Hopper DW, Ghan R, Schlauch KA, Cramer GR. Transcriptomic network analyses of leaf dehydration responses identify highly connected $A B A$ and ethylene signaling hubs in three grapevine species differing in drought tolerance. BMC Plant Biol. 2016;16. doi:10.1186/s12870-016-0804-6.

137. Ghan R, Van Sluyter SC, Hochberg U, Degu A, Hopper DW, Tillet RL, et al. Five omic technologies are concordant in differentiating the biochemical characteristics of the berries of five grapevine (Vitis vinifera L.) cultivars. BMC Genomics. 2015;16:946.

138. Walter J, Nagy L, Hein R, Rascher U, Beierkuhnlein C, Willner E, et al. Do plants remember drought? Hints towards a drought-memory in grasses. Environ Exp Bot. 2011;71:34-40.

139. Kalladan R, Lasky JR, Sharma S, Kumar MN, Juenger TE, Marais DLD, et al. Natural Variation in 9Cis-Epoxycartenoid Dioxygenase 3 and ABA Accumulation. Plant Physiol. 2019;179:1620-31.

140. Liu S, Li M, Su L, Ge K, Li L, Li X, et al. Negative feedback regulation of ABA biosynthesis in peanut (Arachis hypogaea): a transcription factor complex inhibits AhNCED1 expression during water stress. Sci Rep. 2016;6:37943. 
141. Garapati P, Xue G-P, Munné-Bosch S, Balazadeh S. Transcription Factor ATAF1 in Arabidopsis Promotes Senescence by Direct Regulation of Key Chloroplast Maintenance and Senescence Transcriptional Cascades. Plant Physiol. 2015;168:1122-39.

142. Liang C, Cheng S, Zhang Y, Sun Y, Fernie AR, Kang K, et al. Transcriptomic, proteomic and metabolic changes in Arabidopsis thaliana leaves after the onset of illumination. BMC Plant Biol. 2016;16:43.

143. Schwartz SH, Qin X, Zeevaart JAD. Elucidation of the Indirect Pathway of Abscisic Acid Biosynthesis by Mutants, Genes, and Enzymes. Plant Physiol. 2003;131:1591-601.

144. Mergner J, Frejno M, List M, Papacek M, Chen X, Chaudhary A, et al. Mass-spectrometry-based draft of the Arabidopsis proteome. Nature. 2020;579:409-14.

145. Mergner J, Frejno M, Messerer M, Lang D, Samaras P, Wilhelm M, et al. Proteomic and transcriptomic profiling of aerial organ development in Arabidopsis. Sci Data. 2020;7:334.

146. Cruz ER, Nguyen H, Nguyen T, Wallace IS. Functional analysis tools for post-translational modification: a post-translational modification database for analysis of proteins and metabolic pathways. Plant J. 2019;99:1003-13.

147. Heazlewood JL, Durek P, Hummel J, Selbig J, Weckwerth W, Walther D, et al. PhosPhAt: a database of phosphorylation sites in Arabidopsis thaliana and a plant-specific phosphorylation site predictor. Nucleic Acids Res. 2008;36 suppl_1:D1015-21.

148. Potenza E, Racchi ML, Sterck L, Coller E, Asquini E, Tosatto SCE, et al. Exploration of alternative splicing events in ten different grapevine cultivars. BMC Genomics. 2015;16. doi:10.1186/s12864015-1922-5.

149. Vitulo N, Forcato C, Carpinelli EC, Telatin A, Campagna D, D'Angelo M, et al. A deep survey of alternative splicing in grape reveals changes in the splicing machinery related to tissue, stress condition and genotype. BMC Plant Biol. 2014;14.

150. Albacete A, Martínez-Andújar C, Martínez-Pérez A, Thompson AJ, Dodd IC, Pérez-Alfocea F. Unravelling rootstockxscion interactions to improve food security. J Exp Bot. 2015;66:2211-26.

151. Barrios-Masias FH, Knipfer T, Walker MA, McElrone AJ. Differences in hydraulic traits of grapevine rootstocks are not conferred to a common Vitis vinifera scion. Funct Plant Biol. 2019;46:228-35.

152. Kundariya H, Yang X, Morton K, Sanchez R, Axtell MJ, Hutton SF, et al. MSH1-induced heritable enhanced growth vigor through grafting is associated with the RdDM pathway in plants. Nat Commun. 2020;11:1-14.

153. Cookson SJ, Clemente Moreno MJ, Hevin C, Nyamba Mendome LZ, Delrot S, Trossat-Magnin C, et al. Graft union formation in grapevine induces transcriptional changes related to cell wall modification, wounding, hormone signalling, and secondary metabolism. J Exp Bot. 2013;64:2997-3008.

154. Barrs HD, Weatherley PE. A Re-Examination of the Relative Turgidity Technique for Estimating Water Deficits in Leaves. Aust J Biol Sci. 1962;15:413-28.

155. Bolger AM, Lohse M, Usadel B. Trimmomatic: a flexible trimmer for Illumina sequence data. Bioinformatics. 2014;30:2114-20. 
156. Babraham Bioinformatics. Babraham Bioinformatics - FastQC A Quality Control tool for High Throughput Sequence Data. FastQC. 2010.

https://www.bioinformatics.babraham.ac.uk/projects/fastqc/.

157. Jaillon O, Aury, Jean-Marc, Noel, Benjamin, Policriti, Alberto, Clepet, Christian, Casagrande, Alberto, et al. The grapevine genome sequence suggests ancestral hexaploidization in major angiosperm phyla. Nature. 2007;449:463-7.

158. Girollet N, Rubio B, Lopez-Roques C, Valière S, Ollat N, Bert P-F. De novo phased assembly of the Vitis riparia grape genome. Sci Data. 2019;6:1-8.

159. Zhou Y, Minio A, Massonnet M, Solares E, Lv Y, Beridze T, et al. The population genetics of structural variants in grapevine domestication. Nat Plants. 2019;5:965-79.

160. Vondras AM, Minio A, Blanco-Ulate B, Figueroa-Balderas R, Penn MA, Zhou Y, et al. The genomic diversification of grapevine clones. BMC Genomics. 2019;20:972.

161. Minio A, Massonnet M, Figueroa-Balderas R, Castro A, Cantu D. Diploid Genome Assembly of the Wine Grape Carménère. G3 GenesGenomesGenetics. 2019;9:1331-7.

162. Massonnet M, Cochetel N, Minio A, Vondras AM, Muyle A, Lin J, et al. The genetic basis of sex determination in grapevines ( Vitis spp. ). preprint. Evolutionary Biology; 2019. doi:10.1101/2019.12.11.861377.

163. Kim D, Langmead B, Salzberg SL. HISAT: a fast spliced aligner with low memory requirements. Nat Methods. 2015;12:357-60.

164. featureCounts: an efficient general purpose program for assigning sequence reads to genomic features | Bioinformatics | Oxford Academic.

https://academic.oup.com/bioinformatics/article/30/7/923/232889. Accessed 18 May 2020.

165. Love MI, Huber W, Anders S. Moderated estimation of fold change and dispersion for RNA-seq data with DESeq2. Genome Biol. 2014;15. doi:10.1186/s13059-014-0550-8.

166. Gu Z, Eils R, Schlesner M. Complex heatmaps reveal patterns and correlations in multidimensional genomic data. Bioinforma Oxf Engl. 2016;32:2847-9.

167. Galili T. dendextend: an R package for visualizing, adjusting and comparing trees of hierarchical clustering. Bioinformatics. 2015;31:3718-20.

168. Gu Z, Gu L, Eils R, Schlesner M, Brors B. circlize implements and enhances circular visualization in R. Bioinformatics. 2014;30:2811-2.

169. Alexa A, Rahnenführer J, Lengauer T. Improved scoring of functional groups from gene expression data by decorrelating GO graph structure. Bioinformatics. 2006;22:1600-7.

170. Wessel D, Flügge Ul. A method for the quantitative recovery of protein in dilute solution in the presence of detergents and lipids. Anal Biochem. 1984;138:141-3.

171. Minio A, Massonnet M, Vondras A, Figueroa-Balderas R, Blanco-Ulate B, Cantu D. Iso-Seq allows genome-independent transcriptome profiling of grape berry development. preprint. Genomics; 2018. doi:10.1101/269530. 
172. Fox J. An R Companion to Applied Regression. Third. Thousand Oaks CA: Sage; 2020. https://socialsciences.mcmaster.ca/jfox/Books/Companion/.

173. R Core Team. R: The R Project for Statistical Computing. 2018. https://www.r-project.org/.

Figures

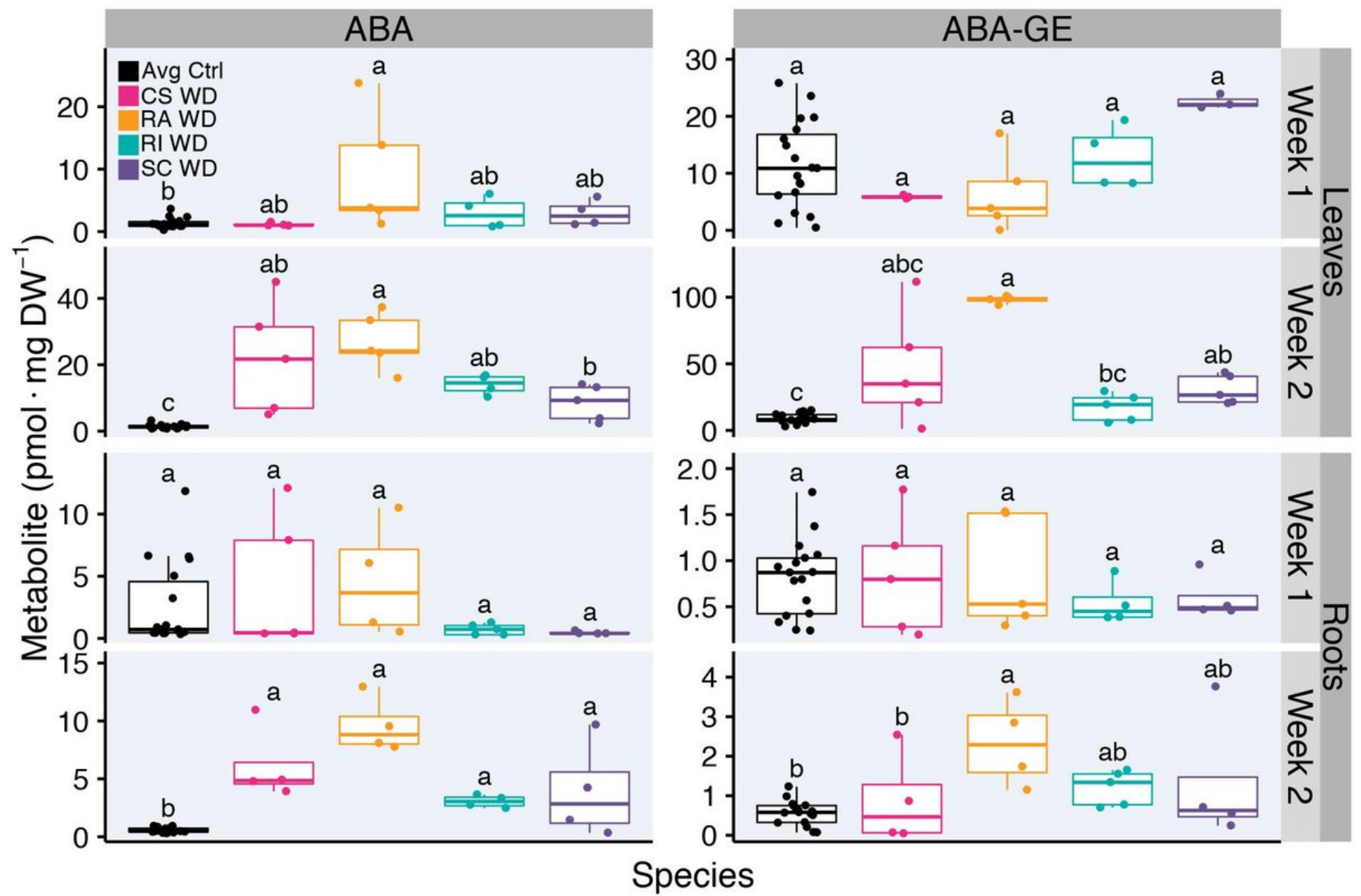

Figure 1

$A B A$ and $A B A-G E$ concentrations after one- and two-weeks of moderate WD. ABA (left) and ABA-GE (right) concentrations (pmol-mg DW-1) in leaves (top) and roots (bottom) after one- and two-weeks of Control (Ctrl) or WD treatment. Control represented for all species per organ per time; there was no significant difference between Control species per organ per week. Two-way ANOVA Tukey's HSD ( $\leq 0.05)$ letters for each metabolite per organ per week. Black corresponds the Control values for all species. Pink, orange, blue and purple correspond to CS, RA, RI, and SC respectively. Each point represents an individual measurement. $n=$ three-five individual vines . 


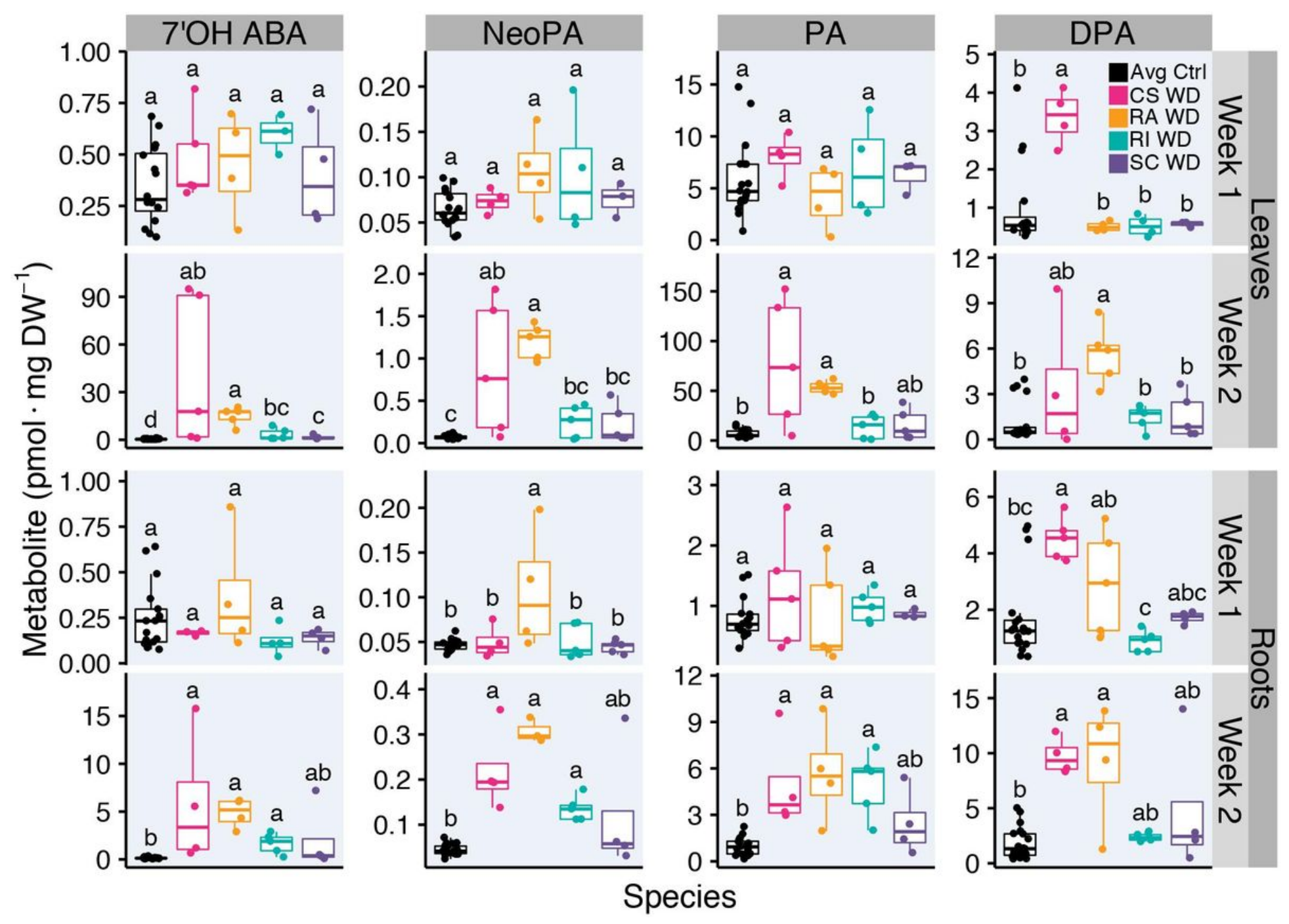

Figure 2

ABA catabolite concentrations after one- and two-weeks of moderate WD. 7'OH ABA, NeoPA, PA, and DPA (left to right) concentrations (pmol.mg DW-1) in leaves (top) and roots (bottom) after one- and two-weeks of Control (Ctrl) or WD treatment. Control represented for all species per organ per time; there was no significant difference between Controls per species per organ per week. Two-way ANOVA Tukey's HSD ( $p$ $\leq 0.05$ ) letters for each metabolite per organ per week. Black corresponds the Control values for all species. Pink, orange, blue and purple correspond to CS, RA, RI, and SC respectively. Each point represents an individual measurement. $\mathrm{n}=$ three-five individual vines. 
UDP-glucose

glucosyltransferases

ß-d-glucosidases

7'-Hydroxy Abscisic Acid

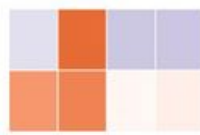

8'-Hydroxy Abscisic Acid

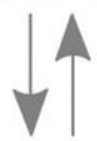

Phaseic Acid
Abscisic Acid
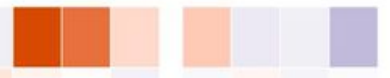

Abscisic acid hydroxylases
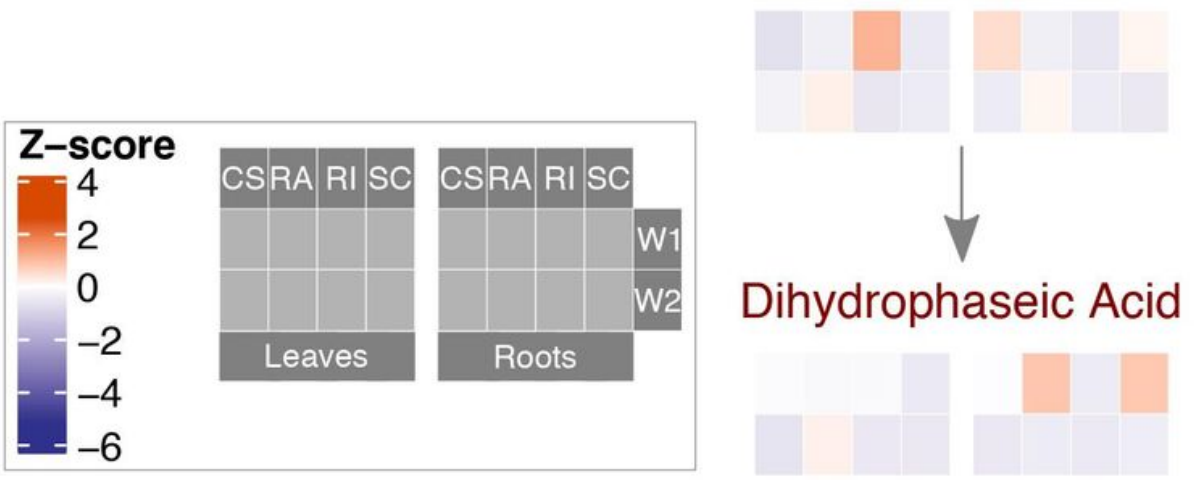

\section{Figure 3}

Average relative ABA metabolite concentrations after moderate WD. Heatmap representation of the average ratio of $A B A$ box-cox transformed metabolite concentrations (WD:C) in leaves (left) and roots (right) after one- (top) and two-weeks (bottom) treatment in the four Vitis species (CS, RA, RI, SC from left to right per organ). Z-scores calculated for all metabolites and conditions as a group with red corresponding to higher and blue corresponding to lower scores. ABA metabolites in red and corresponding genes in grey. Cellular location indicated in black. 


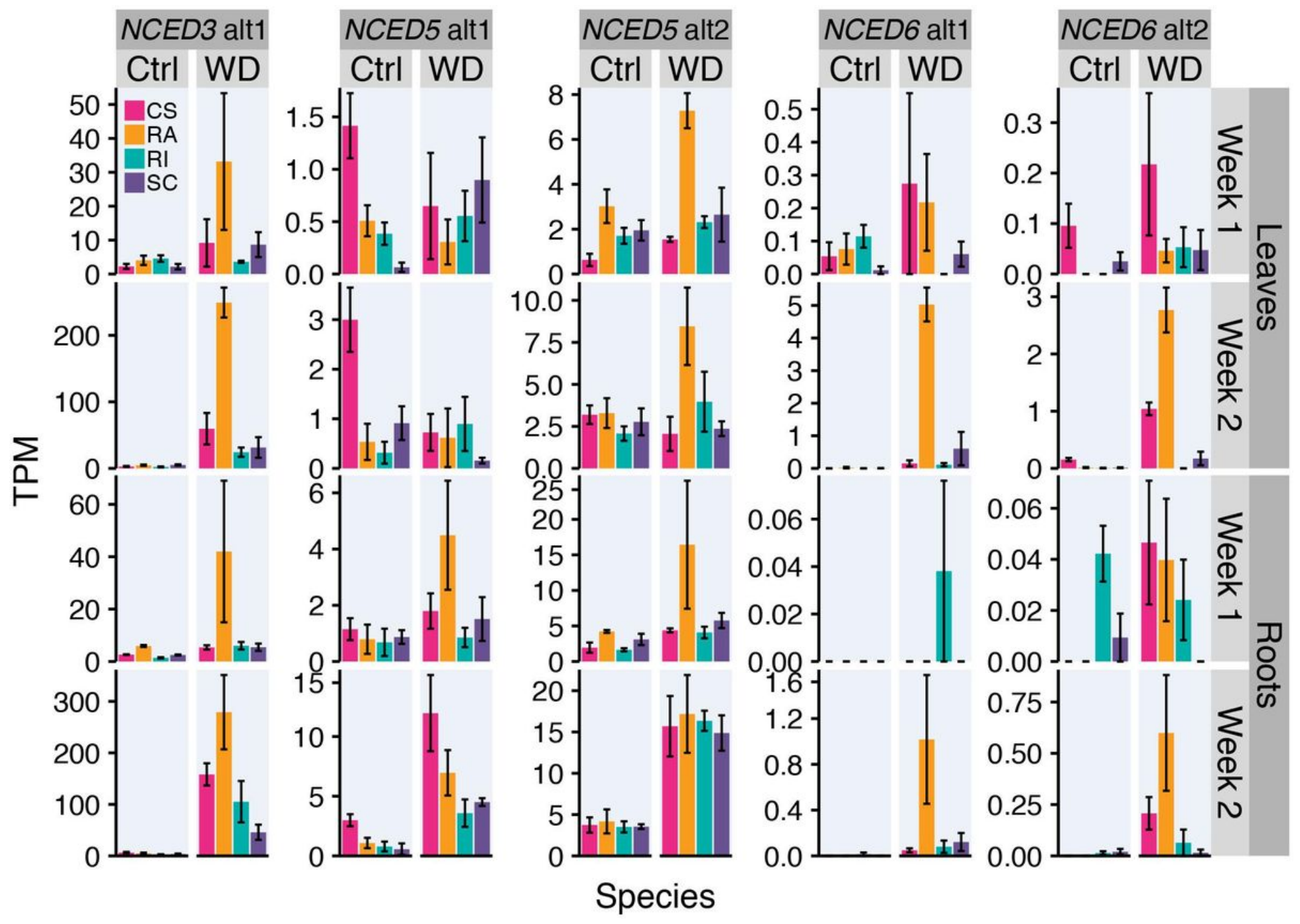

Figure 4

NCED transcript abundance after one- and two-weeks of moderate WD. NCED3, NCED5 alt1 and alt2, NCED6 alt1 and alt2 (left to right) expression represented as TPM after one- (top) and two-weeks (bottom) of Control (Ctrl) (left) or WD (right) treatment in leaves (top) and roots (bottom). Pink, orange, blue and purple correspond to CS, RA, RI, and SC respectively. Mean $\pm S E, n=$ three-five individual vines. 


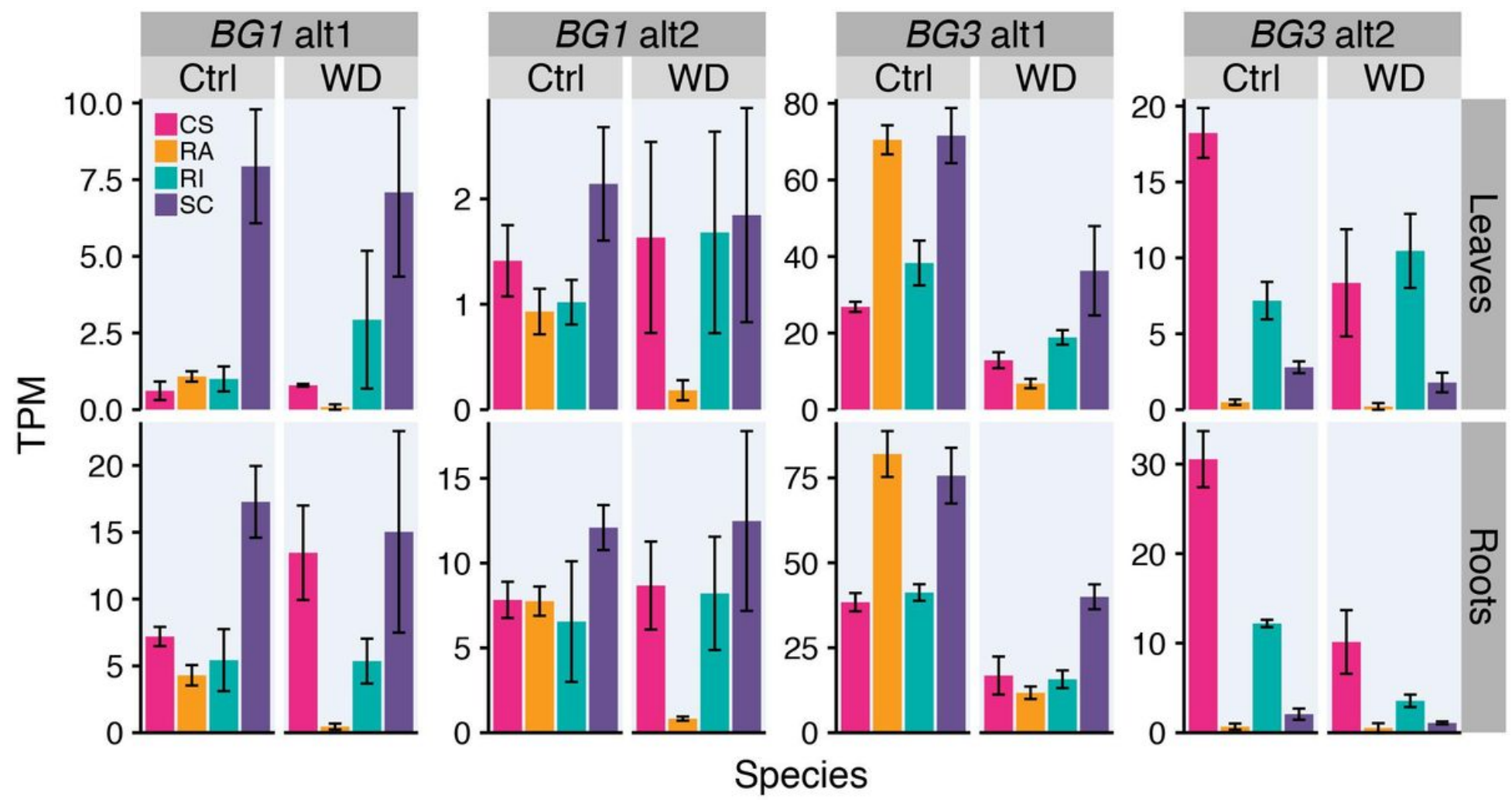

Figure 5

BG1 and BG3 transcript abundance after one- and two-weeks of moderate WD. BG1 alt1 and alt2 and BG3 alt1 and alt2 (left to right) expression represented as TPM after two-weeks of Control (Ctrl) (left) or WD (right) treatment in leaves (top) and roots (bottom). Pink, orange, blue and purple correspond to CS, $\mathrm{RA}, \mathrm{RI}$, and $\mathrm{SC}$ respectively. Mean $\pm \mathrm{SE}, \mathrm{n}=$ three-five individual vines. 

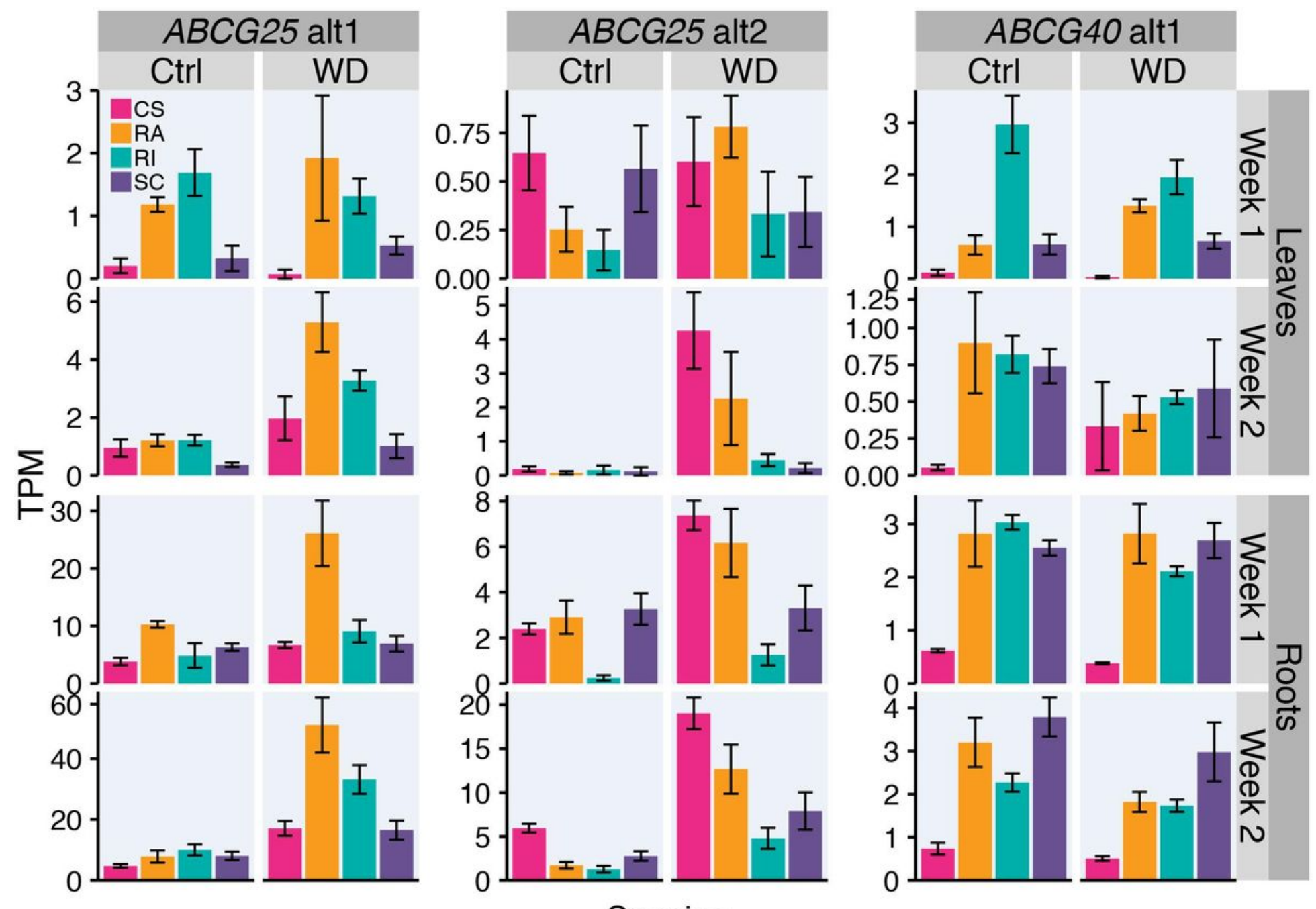

Species

Figure 6

ABCG transcript abundance after one- and two-weeks of moderate WD. ABCG25 alt1 and alt2, and ABCG40 alt1 (left to right) expression represented as TPM after one- (top) and two-weeks (bottom) of Control (Ctrl) (left) or WD (right) treatment in leaves (top) and roots (bottom). Pink, orange, blue and purple correspond to $C S, R A, R I$, and SC respectively. Mean $\pm S E, n=$ three-five individual vines. 

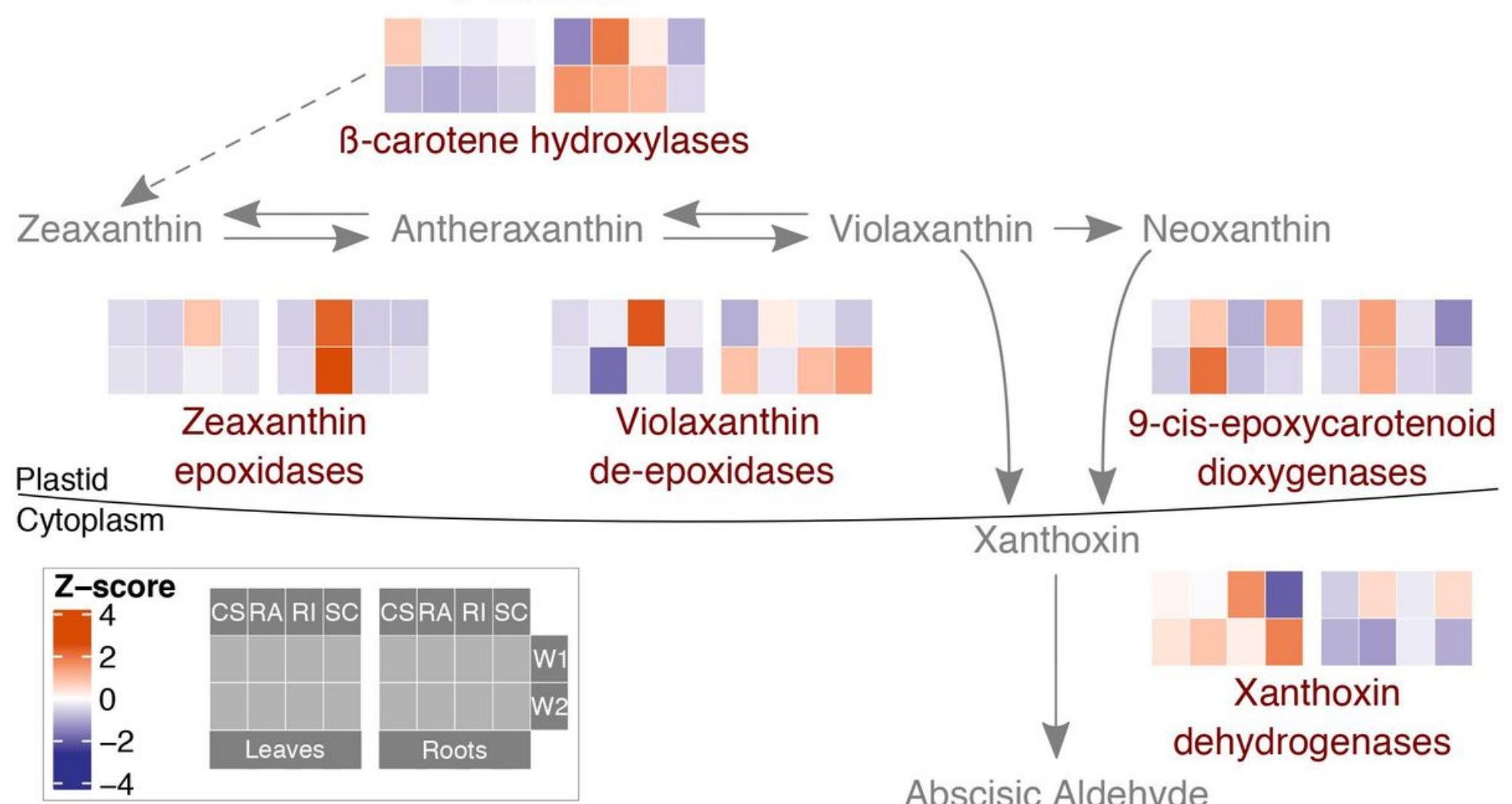

\section{Abscisic Aldehyde}

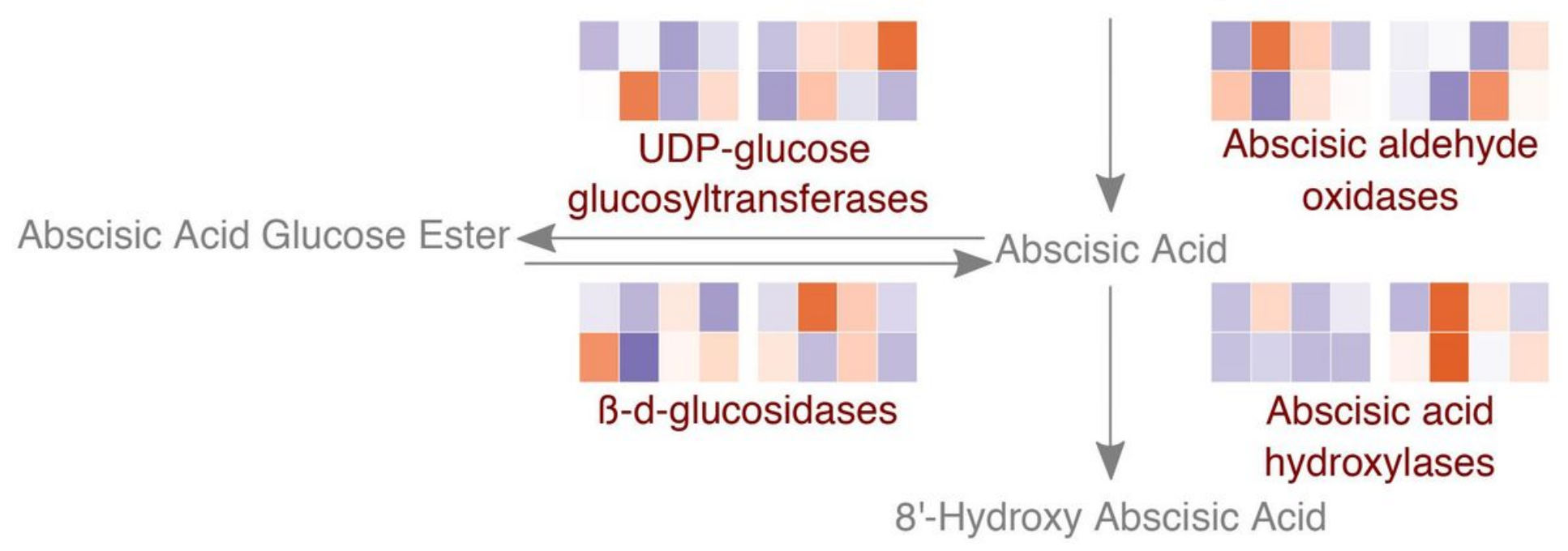

Figure 7

Average relative ABA metabolite gene transcript abundance after moderate WD. Heatmap representation of the average ratio of log2 box-cox transformed ABA metabolite gene TPM (WD:C) in leaves (left) and roots (right) after one- (top) and two-weeks (bottom) treatment in the four Vitis species (CS, RA, RI, SC from left to right per organ). Z-scores calculated for all per gene group (average of multiple paralogs) for all conditions with red corresponding to higher and blue corresponding to lower scores. ABA metabolite genes in red and resultant metabolites in grey. Cellular location indicated in black. 


\begin{tabular}{|c|c|c|c|c|c|c|c|c|c|c|c|c|c|c|}
\hline MElightcyan ( $n=298$ ) & $0.25(0.05)$ & $-0.071(0.6)$ & $-0.024(0.9)$ & $-0.16(0.2) \quad 0$ & $0.63(3 e-08)-c$ & $-0.63(3 e-08)$ & $0.14(0.3)$ & $-0.14(0.3)$ & $-0.23(0.07)-$ & $-0.35(0.006)-$ & $-0.23(0.08)$ & $0.07(0.6)$ & $-0.25(0.05)$ & $-0.22(0.09)$ \\
\hline MEroyalblue $(n=180)$ & $-0.68(9 e-10)$ & $0.23(0.07)$ & $0.25(0.05)$ & $0.24(0.06)$ & $0.43(6 e-04)-c$ & $-0.43(6 e-04)$ & $0.026(0.8)$ & $-0.026(0.8)$ & $0.051(0.7)$ & $0.0012(1)$ & $0.14(0.3)$ & $-0.35(0.006)$ & $0.008(1)$ & $-0.12(0.4)$ \\
\hline MEturquoise $(n=3728)$ & $-0.61(2 e-07)$ & $0.13(0.3)$ & $0.44(3 e-04)$ & $0.084(0.5)$ & $0.066(0.6)$ & $-0.066(0.6)$ & $0.084(0.5)$ & $-0.084(0.5)$ & $-0.049(0.7)$ & $0.0048(1)$ & $-0.033(0.8)$ & $-0.4(0.001)$ & $-0.0065(1)$ & $-0.18(0.2)$ \\
\hline MEyellow ( $n=2071)$ & $-0.57(1 e-06)$ & $0.15(0.3)$ & $0.087(0.5)$ & $0.35(0.005)$ & $0.36(0.004)-$ & $-0.36(0.004)$ & $0.047(0.7)$ & $-0.047(0.7)$ & $-0.27(0.04)$ & $-0.2(0.1)$ & $-0.19(0.1)=-$ & $-0.41(8 \mathrm{e}-04)$ & $-0.15(0.3)$ & $-0.35(0.005)$ \\
\hline MEmidnightblue ( $\mathrm{n=298)}$ & $-0.6(2 e-07)-$ & $-0.46(1 e-04)$ & $0.62(1 \mathrm{e}-07)$ & $0.49(6 e-05)$ & $-0.039(0.8)$ & $0.039(0.8)$ & $0.0013(1)$ & $-0.0013(1)$ & $-0.078(0.5)$ & $-0.099(0.4)$ & $-0.047(0.7)$ & $-0.37(0.003)$ & $-0.11(0.4)$ & $-0.15(0.2)$ \\
\hline MEpurple $(n=696)$ & $-0.44(4 e-04)$ & $-0.09(0.5)$ & $0.72(6 e-11)$ & $-0.13(0.3)$ & $0.044(0.7)$ & $-0.044(0.7)$ & $0.17(0.2)$ & $-0.17(0.2)$ & $-0.15(0.2)$ & $-0.16(0.2)-$ & $-0.18(0.2)$ & $-0.45(3 e-04)$ & $-0.12(0.3)$ & $-0.29(0.02)$ \\
\hline MEdarkred ( $n=151)$ & $0.13(0.3)$ & $0.32(0.01)$ & $-0.93(7 \mathrm{e}-28)$ & $0.41(0.001)$ & $0.0082(0.9)-$ & $-0.0082(0.9)$ & $-0.081(0.5)$ & $0.081(0.5)$ & $0.13(0.3)$ & $0.15(0.3)$ & $0.25(0.05)$ & $0.18(0.2)$ & $0.16(0.2)$ & $0.14(0.3)$ \\
\hline MEpink ( $n=911$ ) & $-0.59(4 e-07)$ & $-0.14(0.3)$ & $-0.23(0.07)$ & $0.94(5 e-30)$ & $-0.0032(1)$ & $0.0032(1)$ & $-0.024(0.9)$ & $0.024(0.9)$ & $-0.017(0.9)$ & $0.0019(1)$ & $0.14(0.3)$ & $-0.29(0.02)$ & $0.0078(1)$ & $-0.07(0.6)$ \\
\hline MEorange ( $n=98$ ) & $-0.75(2 e-12)$ & $0.78(8 e-14)$ & $-0.23(0.08)$ & $0.21(0.1)$ & $0.026(0.8)$ & $-0.026(0.8)$ & $-0.091(0.5)$ & $0.091(0.5)$ & $0.31(0.02)$ & $0.39(0.002) 0$ & $0.41(8 \mathrm{e}-04)$ & $-0.29(0.02)$ & $0.37(0.003)$ & $0.11(0.4)$ \\
\hline MEskyblue ( $n=63$ ) & $-0.38(0.002)$ & $0.33(0.01)$ & $-0.33(0.009) 0$ & $0.37(0.003)$ & $0.11(0.4)$ & $-0.11(0.4)$ & $0.2(0.1)$ & $-0.2(0.1)$ & $0.19(0.1)$ & $0.14(0.3)$ & $0.27(0.03)$ & $-0.048(0.7)$ & $0.26(0.04)$ & $0.096(0.5)$ \\
\hline MEblack $(n=1134)$ & $0.017(0.9)$ & $-0.13(0.3)$ & $-0.36(0.004)$ & $0.44(4 \mathrm{e}-04)$ & $0.37(0.003)-$ & $-0.37(0.003)$ & $0.077(0.6)$ & $-0.077(0.6)$ & $-0.39(0.002)-$ & $-0.36(0.004)-$ & $-0.31(0.01)$ & $-0.099(0.4)$ & $-0.28(0.03)-$ & $-0.34(0.007)$ \\
\hline MEblue $(n=2990)$ & $-0.048(0.7)$ & $0.067(0.6)$ & $-0.088(0.5)$ & $0.064(0.6)$ & $0.61(1 \mathrm{e}-07)-c$ & $-0.61(1 e-07)$ & $0.11(0.4)$ & $-0.11(0.4)$ & $-0.45(2 e-04)-$ & $-0.44(3 e-04)-$ & $-0.39(0.002)$ & $-0.17(0.2)$ & $-0.35(0.006)-$ & $-0.44(3 e-04)$ \\
\hline MEdarkturquoise $(n=130)$ & $0.19(0.1)$ & $-0.91(4 e-25)$ & $0.21(0.1)$ & $0.5(3 e-05)$ & $0.081(0.5)$ & $-0.081(0.5)$ & $0.022(0.9)$ & $-0.022(0.9)$ & $-0.34(0.008)-$ & $-0.44(3 e-04)-$ & $-0.33(0.009)$ & $0.00093(1)$ & $-0.37(0.003)$ & $-0.22(0.09)$ \\
\hline MEgrey60 ( $n=297)$ & $0.35(0.005)$ & $-0.56(2 e-06)-$ & $-0.58(1 e-06)$ & $0.72(6 e-11)$ & $-0.052(0.7)$ & $0.052(0.7)$ & $-0.016(0.9)$ & $0.016(0.9)$ & $-0.17(0.2)$ & $-0.23(0.07)$ & $-0.085(0.5)$ & $0.2(0.1)$ & $-0.18(0.2)$ & $-0.02(0.9)$ \\
\hline MEdarkgreen $(n=148)$ & $-0.11(0.4)$ & $-0.055(0.7)$ & $0.19(0.1)$ & $-0.018(0.9)-(r-r$ & $-0.48(9 e-05) 0$ & $0.48(9 e-05)-$ & $-0.39(0.002)$ & $0.39(0.002)$ & $0.45(2 e-04)$ & $0.44(4 \mathrm{e}-04)$ & $0.4(0.001)$ & $0.061(0.6)$ & $0.27(0.03)$ & $0.43(5 e-04)$ \\
\hline MElightyellow ( $\mathrm{n=275)}$ & $-0.075(0.6)$ & $0.14(0.3)$ & $-0.12(0.3)$ & $0.057(0.7)$ & $-0.67(2 e-09) 0$ & $0.67(2 e-09)-$ & $-0.34(0.007)$ & $0.34(0.007)$ & $0.64(2 e-08)$ & $0.64(2 \mathrm{e}-08) 0$ & $0.62(7 e-08)$ & $0.23(0.07)$ & $0.51(2 e-05)$ & $0.6(2 e-07)$ \\
\hline MEred $(n=1935)$ & $-0.23(0.07)$ & $0.45(3 e-04)$ & $0.59(4 e-07)-$ & $-0.74(5 e-12)$ & $0.084(0.5)$ & $-0.084(0.5)$ & $0.044(0.7)$ & $-0.044(0.7)$ & $0.029(0.8)$ & $0.081(0.5)$ & $-0.075(0.6)$ & $-0.24(0.06)$ & $0.063(0.6)$ & $-0.12(0.3)$ \\
\hline MEdarkgrey ( $n=99)$ & $-0.48(8 e-05)$ & $0.45(3 e-04)$ & $0.17(0.2)$ & $-0.1(0.4)$ & $-0.23(0.07)$ & $0.23(0.07)$ & $0.026(0.8)$ & $-0.026(0.8)$ & $0.32(0.01)$ & $0.28(0.03)$ & $0.35(0.005)$ & $-0.26(0.04)$ & $0.29(0.02)$ & $0.12(0.4)$ \\
\hline MEbrown $(n=2261)$ & $-0.22(0.09)$ & $0.11(0.4)$ & $0.45(3 e-04)$ & $-0.3(0.02)$ & $-0.38(0.003) 0$ & $0.38(0.003)$ & $-0.15(0.2)$ & $0.15(0.2)$ & $0.47(1 \mathrm{e}-04)$ & $0.4(0.001)$ & $0.4(0.001)$ & $-0.013(0.9)$ & $0.33(0.008)$ & $0.33(0.009)$ \\
\hline MEgreen $(n=2009)$ & $-0.79(2 e-14)$ & $0.3(0.02)$ & $0.38(0.003)$ & $0.16(0.2)$ & $-0.25(0.05)$ & $0.25(0.05)$ & $-0.16(0.2)$ & $0.16(0.2)$ & $0.47(1 \mathrm{e}-04)$ & $0.46(2 \mathrm{e}-04) 0$ & $0.49(5 e-05)$ & $-0.27(0.03)$ & $0.38(0.002)$ & $0.24(0.06)$ \\
\hline MElightgreen $(n=296)$ & $0.43(4 e-04)$ & $-0.46(2 e-04)$ & $0.66(6 e-09)-$ & $-0.59(4 e-07)$ & $0.016(0.9)$ & $-0.016(0.9)$ & $0.049(0.7)$ & $-0.049(0.7)$ & $-0.22(0.09)$ & $-0.29(0.02)-($ & $-0.39(0.002)$ & $0.068(0.6)$ & $-0.25(0.05)$ & $-0.16(0.2)$ \\
\hline MEdarkorange $(n=93)$ & $0.36(0.004)$ & $-0.18(0.2)$ & $0.086(0.5)$ & $-0.26(0.04)$ & $0.18(0.2)$ & $-0.18(0.2)$ & $0.056(0.7)$ & $-0.056(0.7)$ & $-0.3(0.02)$ & $-0.27(0.03)-$ & $-0.35(0.006)$ & $-0.014(0.9)$ & $-0.24(0.06)$ & $-0.25(0.05)$ \\
\hline MEtan $(n=548)$ & $0.4(0.001)$ & $-0.13(0.3)$ & $0.11(0.4)$ & $-0.38(0.003)$ & $0.061(0.6)$ & $-0.061(0.6)$ & $0.026(0.8)$ & $-0.026(0.8)$ & $-0.25(0.05)$ & $-0.25(0.05)$ & $-0.34(0.007)$ & $0.1(0.4)$ & $-0.22(0.09)$ & $-0.19(0.1)$ \\
\hline MEmagenta $(n=780)$ & $0.81(3 e-15)$ & $-0.049(0.7)$ & $-0.15(0.3)$ & $-0.62(6 e-08)$ & $0.21(0.1)$ & $-0.21(0.1)$ & $0.12(0.4)$ & $-0.12(0.4)$ & $-0.28(0.03)$ & $-0.3(0.02)-$ & $-0.38(0.002)$ & $0.3(0.02)$ & $-0.23(0.07)$ & $-0.16(0.2)$ \\
\hline MEwhite $(n=63)$ & $0.73(2 e-11)$ & $-0.26(0.04)$ & $-0.29(0.02)$ & $-0.22(0.09)$ & $0.079(0.5)$ & $-0.079(0.5)$ & $0.034(0.8)$ & $-0.034(0.8)$ & $-0.077(0.5)$ & $-0.15(0.3)$ & $-0.13(0.3)$ & $0.43(5 e-04)$ & $-0.1(0.4)$ & $0.086(0.5)$ \\
\hline \multirow[t]{2}{*}{ MEgrey (n=12789) } & $0.97(1 e-37)$ & $-0.36(0.004)$ & $-0.33(0.01)$ & $-0.33(0.01)$ & $0.057(0.7)$ & $-0.057(0.7)$ & $0.0065(1)$ & $-0.0065(1)$ & $-0.21(0.1)$ & $-0.28(0.03)$ & $-0.3(0.02)$ & $0.46(2 e-04)$ & $-0.22(0.09)$ & $-0.029(0.8)$ \\
\hline & & & & & & & & & & & & & & \\
\hline
\end{tabular}

\section{Figure 8}

WGCNA of the leaves. Heatmap representation of the association of modules and experimental conditions for the leaf samples. Experimental conditions listed in columns and modules in rows. Module eigengene Pearson's correlation coefficient and p-value (in parenthesis) listed for each module per each experimental condition. Cell colors range from high correlation (red) to negative correlation (blue). Number of genes in each module represented by $\mathrm{n}$ in parenthesis after module name. 


\begin{tabular}{|c|c|c|c|c|c|c|c|c|c|c|c|c|c|c|}
\hline MEsaddlebrown $(n=170)$ & $-0.065(0.6)$ & $0.022(0.9)$ & $0.26(0.05)$ & $-0.2(0.1)$ & $-0.28(0.04)$ & $0.28(0.04)$ & $0.077(0.6)$ & $-0.077(0.6)$ & $0.11(0.4)$ & $0.054(0.7)$ & $-0.09(0.5)$ & $-0.1(0.4)$ & $0.035(0.8)$ & $-0.037(0.8)$ \\
\hline MEwhite $(n=213)$ & $0.2(0.1)$ & $-0.09(0.5)$ & $-0.38(0.004)$ & $0.25(0.06)$ & $-0.74(5 e-11)$ & $0.74(5 e-11)$ & $-0.076(0.6)$ & $0.076(0.6)$ & $0.33(0.01)$ & $0.2(0.1)$ & $0.17(0.2)$ & $0.34(0.01)$ & $0.3(0.02)$ & $0.27(0.04)$ \\
\hline MEdarkolivegreen $(n=47)$ & $0.11(0.4)$ & $-0.14(0.3)$ & $0.12(0.4)$ & $-0.088(0.5)-$ & $-0.49(1 e-04)$ & $0.49(1 e-04)$ & $0.23(0.09)$ & $-0.23(0.09)$ & $-0.096(0.5)$ & $-0.17(0.2)$ & $-0.3(0.02)$ & $-0.15(0.3)$ & $-0.12(0.4)$ & $-0.19(0.2)$ \\
\hline MEdarkturquoise $(n=333)$ & $0.011(0.9)$ & $-0.3(0.03)$ & $-0.18(0.2)$ & $0.46(3 e-04)$ & $-0.35(0.008)$ & $0.35(0.008)$ & $0.33(0.01)$ & $-0.33(0.01)$ & $-0.22(0.1)$ & $-0.34(0.01)$ & $-0.46(3 e-04)$ & $-0.34(0.009)$ & $-0.19(0.1)$ & $-0.37(0.005)$ \\
\hline MEdarkorange $(n=230)$ & $-0.66(2 e-08)$ & $0.55(1 e-05)-$ & $-0.46(4 e-04)$ & $0.59(1 e-06)$ & $0.037(0.8)$ & $-0.037(0.8)$ & $0.1(0.4)$ & $-0.1(0.4)$ & $-0.07(0.6)$ & $-0.021(0.9)$ & $0.0054(1)$ & $-0.22(0.1)$ & 0.0016 (1) & $-0.21(0.1)$ \\
\hline MEsteelblue $(n=101)$ & $-0.28(0.03)$ & $-0.043(0.7)$ & $-0.51(4 e-05)$ & $0.84(2 e-16)$ & $-0.00017(1)$ & 0.00017 & $-0.027(0.8)$ & $0.027(0.8)$ & 0.0011 (1) & $0.047(0.7)$ & $0.014(0.9)$ & $-0.067(0.6)$ & $0.069(0.6)$ & $-0.075(0.6)$ \\
\hline MEturquoise $(n=2173)$ & $-0.96(3 e-32)$ & $0.45(5 e-04)$ & 0.35 & $0.21(0.1)$ & $-0.11(0.4)$ & $0.11(0.4)$ & $-0.087(0.5)$ & $0.087(0.5)$ & $-0.068(0.6)$ & $-0.054(0.7)$ & $0.09(0.5)$ & $-0.22(0.1)$ & $-0.014(0.9)$ & $-0.11(0.4)$ \\
\hline MEred $(n=1511)$ & $-0.88(1 e-19)$ & $0.045(0.7)$ & $0.5(8 e-05)$ & $0.39(0.003)$ & $0.13(0.3)$ & $-0.13(0.3)$ & $0.11(0.4)$ & $-0.11(0.4)$ & $-0.26(0.05)$ & $-0.24(0.07)$ & $-0.2(0.1)$ & $-0.46(3 e-04)$ & $-0.21(0.1)$ & $-0.32(0.01)$ \\
\hline MEyellow ( $n=1763$ ) & $-0.65(3 e-08)$ & 3) $-0.13(0.3)$ & $0.012(0.9)$ & $0.8(7 e-14)$ & $0.12(0.4)$ & $-0.12(0.4)$ & $0.17(0.2)$ & $-0.17(0.2)$ & $-0.24(0.07)$ & $-0.23(0.08)$ & $-0.25(0.06)$ & $-0.43(9 e-04)$ & $-0.16(0.2)$ & $-0.34(0.01)$ \\
\hline MEdarkgreen $(n=357)$ & $-0.51(4 e-05)$ & $5)-0.58(2 e-06)$ & $0.49(1 e-04)$ & $0.65(6 e-08)$ & $0.16(0.2)$ & $-0.16(0.2)$ & $0.12(0.4)$ & $-0.12(0.4)$ & $-0.27(0.04)$ & $-0.37(0.005)$ & $-0.31(0.02)$ & $-0.41(0.001)$ & $-0.21(0.1)$ & $-0.27(0.04)$ \\
\hline MEorange ( $n=269)$ & $0.024(0.9)$ & $-0.92(1 e-24)$ & $0.52(3 e-05)$ & $0.39(0.003)$ & $0.08(0.6)$ & $-0.08(0.6)$ & $0.013(0.9)$ & $-0.013(0.9)$ & $-0.17(0.2)$ & $-0.28(0.03)$ & $-0.28(0.04)$ & $-0.2(0.1)$ & $-0.15(0.3)$ & $-0.094(0.5)$ \\
\hline MEtan $(n=610)$ & $-0.1(0.4)$ & $-0.32(0.02)$ & $-0.22(0.1)$ & $0.64(6 e-08)$ & $0.22(0.1)$ & $-0.22(0.1)$ & $0.45(5 e-04)$ & $-0.45(5 e-0.4)$ & ) $-0.37(0.004)$ & $-0.45(5 e-04)$ & $1)-0.51(6 e-05)-$ & $1)-0.49(1 e-04)$ & $-0.29(0.03)$ & $-0.49(1 e-04)$ \\
\hline MEblack $(n=1065)$ & $0.13(0.3)$ & $-0.36(0.006)$ & $-0.09(0.5)$ & $0.31(0.02)$ & $0.46(3 e-04)-$ & $-0.46(3 e-04)$ & $0.45(5 e-04)$ & $-0.45(5 e-0.4)$ & $-0.43(9 e-04)$ & 4) $-0.4(0.002)$ & $-0.51(6 e-05)-$ & $-0.47(2 e-04)$ & $-0.37(0.004)$ & $-0.48(1 e-04$ \\
\hline MEbrown $(n=1785)$ & $0.28(0.03)$ & $-0.2(0.1)$ & $-0.096(0.5)$ & $-0.0048(1)$ & $0.42(0.001)$ & $-0.42(0.001)$ & $0.51(6 e-05)$ & $-0.51(6 e+05)$ & )$-0.41(0.002)$ & $-0.42(0.001)$ & $-0.53(3 e-05)-$ & )$-0.44(6 e-0$ & $-0.39(0.003)$ & $-0.49(1 e-04$ \\
\hline MEblue $(n=2111)$ & $-0.5(6 e-05)$ & $-0.01(0.9)$ & $0.22(0.1)$ & $0.33(0.01)$ & $0.37(0.005)$ & $-0.37(0.005)$ & $0.49(1 e-04)$ & $-0.49(1 e-0.4)$ & $-0.43(9 e-04)$ & $4)-0.45(5 e-0.4)$ & ()-0.44 (7e-04)- & $-0.61(6 e-07)$ & $-0.36(0.006)$ & $-0.53(2 e-05$ \\
\hline MEdarkgrey $(n=276)$ & $-0.092(0.5)$ & $-0.28(0.04)$ & $0.19(0.2)$ & $0.19(0.2)$ & $0.78(6 e-13)-$ & $-0.78(6 e-13)$ & $0.3(0.02)$ & $-0.3(0.02)$ & $-0.45(5 e-0.4)$ & ) $-0.5(9 e-05)$ & $-0.46(3 e-04)-$ & $-0.53(2 e-05)$ & $-0.38(0.004)$ & $-0.48(2 e-04$ \\
\hline MEviolet $(n=57)$ & $0.34(0.01)$ & $-0.021(0.9)$ & $-0.79(5 e-13)$ & $0.43(7 e-04)$ & $0.23(0.08)$ & $-0.23(0.08)$ & $0.28(0.04)$ & $-0.28(0.04)$ & $-0.18(0.2)$ & $-0.19(0.2)$ & $-0.29(0.03)$ & $-0.18(0.2)$ & $-0.13(0.3)$ & $-0.29(0.03)$ \\
\hline MEgreenyellow ( $n=704$ ) & $0.5(8 e-05)-$ & $-0.56(6 e-06)$ & $-0.58(2 e-06)$ & $0.61(4 e-07)$ & $0.019(0.9)$ & $-0.019(0.9)$ & $-0.026(0.9)$ & $0.026(0.9)$ & $0.095(0.5)$ & $0.034(0.8)$ & $-0.031(0.8)$ & $0.16(0.2)$ & $0.11(0.4)$ & $0.099(0.5)$ \\
\hline MElightyellow $(n=396)$ & $0.17(0.2)$ & $-0.6(9 e-07)$ & $-0.41(0.002)$ & $0.81(1 e-14)$ & $0.15(0.3)$ & $-0.15(0.3)$ & $0.1(0.5)$ & $-0.1(0.5)$ & $-0.15(0.3)$ & $-0.23(0.09)$ & $-0.28(0.03)$ & $-0.17(0.2)$ & $-0.096(0.5)$ & $-0.19(0.2)$ \\
\hline MEsalmon ( $n=603$ ) & $-0.042(0.8)$ & $0.14(0.3)$ & $0.028(0.8)$ & $-0.13(0.3)$ & $-0.28(0.04)$ & $0.28(0.04)$ & $-0.22(0.1)$ & $0.22(0.1)$ & $0.24(0.07)$ & $0.19(0.2)$ & $0.36(0.006)$ & $0.35(0.007)$ & $0.27(0.04)$ & $0.34(0.01)$ \\
\hline MEpaleturquoise $(n=70)$ & $0.0073(1)$ & $0.13(0.4)$ & $0.058(0.7)$ & $-0.19(0.2)$ & $-0.41(0.001)$ & $0.41(0.001)$ & $-0.41(0.002)$ & $0.41(0.002)$ & $0.21(0.1)$ & $0.35(0.008)$ & $0.37(0.005)$ & $0.36(0.007)$ & $0.18(0.2)$ & $0.35(0.007)$ \\
\hline MElightgreen $(n=402)$ & $0.34(0.009)$ & $0.37(0.004)$ & $-0.08(0.6)$ & $-0.65(4 e-08)$ & $-0.29(0.03)$ & $0.29(0.03)$ & $-0.39(0.003)$ & $0.39(0.003)$ & $0.46(3 e-04)$ & $0.49(1 e-04)$ & $0.54(2 e-05)$ & $0.6(8 e-07)$ & $0.37(0.005)$ & $0.53(2 e-05)$ \\
\hline MEpink ( $n=989$ ) & $-0.42(0.001)$ & $0.39(0.003)$ & $0.2(0.1)$ & $-0.15(0.3)$ & $-0.36(0.006)$ & $0.36(0.006)$ & $-0.44(5 e-04)$ & $0.44(5 e-04)$ & $0.36(0.006)$ & $0.38(0.004)$ & $0.53(2 e-05)$ & $0.37(0.005)$ & $0.33(0.01)$ & $0.41(0.001)$ \\
\hline MEmidnightblue ( $n=536)$ & $0.094(0.5)$ & $0.14(0.3)$ & $-0.041(0.8)$ & $-0.2(0.1)$ & $-0.56(6 e-06)$ & $0.56(6 e-06)$ & $-0.5(7 e-05)$ & $0.5(7 e-05)$ & $0.47(2 e-04)$ & $0.44(6 e-04)$ & $0.5(9 e-05)$ & $0.56(7 e-06)$ & $0.41(0.001)$ & $0.53(2 e-05)$ \\
\hline MEroyalblue $(n=382)$ & $0.083(0.5)$ & $0.13(0.3)$ & $-0.18(0.2)$ & $-0.037(0.8)$ & $-0.38(0.003)$ & $0.38(0.003)$ & $-0.52(4 e-05)$ & $0.52(4 e-05)$ & $0.48(1 e-04)$ & $0.54(1 e-05)$ & $0.6(7 e-07)$ & $0.61(6 e-07)$ & $0.43(8 e-04)$ & $0.57(4 e-06)$ \\
\hline MEgreen $(n=1516)$ & $0.87(2 e-18)$ & $-0.17(0.2)$ & $-0.31(0.02)-$ & $-0.44(6 e-04)$ & $0.084(0.5)$ & $-0.084(0.5)$ & $-0.11(0.4)$ & $0.11(0.4)$ & $0.19(0.2)$ & $0.27(0.05)$ & $0.12(0.4)$ & $0.35(0.008)$ & $0.1(0.5)$ & $0.23(0.08)$ \\
\hline MEpurple $(n=740)$ & $0.61(5 e-07)$ & & & ) $-0.6(7 e-07)$ & $-0.051(0.7)$ & $0.051(0.7)$ & $-0.093(0.5)$ & $0.093(0.5)$ & $0.26(0.05)$ & $0.35(0.007)$ & $0.27(0.05)$ & $0.4(0.002)$ & $0.19(0.2)$ & $0.24(0.07)$ \\
\hline MEskyblue ( $n=196$ ) & $0.46(3 e-04)$ & $0.39(0.003)$ & $-0.37(0.005)-$ & $-0.51(5 e-05)$ & $0.32(0.01)$ & $-0.32(0.01)$ & $0.24(0.07)$ & $-0.24(0.07)$ & $-0.18(0.2)$ & $-0.077(0.6)$ & $-0.21(0.1)$ & $-0.13(0.4)$ & $-0.2(0.1)$ & $-0.24(0.07)$ \\
\hline MEcyan $(n=553)$ & $-0.14(0.3)$ & $0.4(0.002)$ & $0.16(0.2)$ & $-0.41(0.001)$ & $0.026(0.8)$ & $-0.026(0.8)$ & $-0.15(0.3)$ & $0.15(0.3)$ & $0.0064(1)$ & $0.15(0.3)$ & $0.023(0.9)$ & $-0.0086(0.9)$ & $-0.052(0.7)$ & $-0.031(0.8)$ \\
\hline MEgrey60 $(n=517)$ & $0.22(0.1)$ & $0.14(0.3)$ & $0.38(0.003)$ & $-0.75(3 e-11)$ & $0.33(0.01)$ & $-0.33(0.01)$ & $0.21(0.1)$ & $-0.21(0.1)$ & $-0.24(0.07)$ & $-0.16(0.2)$ & $-0.26(0.05)$ & $-0.24(0.07)$ & $-0.29(0.03)$ & $-0.26(0.06)$ \\
\hline MElightcyan $(n=534)$ & $0.54(1 e-05)$ & $-0.17(0.2)$ & $0.49(1 e-04)$ & $-0.87(3 e-18)$ & $0.073(0.6)$ & $-0.073(0.6)$ & $-0.05(0.7)$ & $0.05(0.7)$ & $0.028(0.8)$ & $0.065(0.6)$ & $0.011(0.9)$ & $0.14(0.3)$ & $-0.052(0.7)$ & $0.13(0.3)$ \\
\hline MEdarkred $(n=358)$ & $0.006(1)$ & $0.42(0.001)$ & $0.5(7 e-05)$ & $-0.92(9 e-24)$ & $-0.024(0.9)$ & $0.024(0.9)$ & $-0.14(0.3)$ & $0.14(0.3)$ & $0.083(0.5)$ & $0.17(0.2)$ & $0.19(0.2)$ & $0.13(0.4)$ & $0.016(0.9)$ & $0.13(0.3)$ \\
\hline MEmagenta $(n=915)$ & $-0.55(1 e-05)$ & $0.29(0.03)$ & $0.77(3 e-12)-$ & $-0.47(2 e-04)$ & $0.12(0.4)$ & $-0.12(0.4)$ & $0.065(0.6)$ & $-0.065(0.6)$ & $-0.19(0.2)$ & $-0.12(0.4)$ & $-0.091(0.5)$ & $-0.3(0.03)$ & $-0.2(0.1)$ & $-0.2(0.1)$ \\
\hline \multirow[t]{2}{*}{ MEgrey $(n=12105)$} & $1(3 e-74)$ & $-0.36(0.006)$ & $-0.33(0.01)$ & $-0.36(0.006)$ & $0.03(0.8)$ & $-0.03(0.8)$ & $-0.055(0.7)$ & $0.055(0.7)$ & $0.16(0.2)$ & $0.14(0.3)$ & $0.039(0.8)$ & $0.35(0.008)$ & $0.094(0.5)$ & $0.23(0.09)$ \\
\hline & & & & & & & & & & & & & & \\
\hline
\end{tabular}

\section{Figure 9}

WGCNA of the roots. Heatmap representation of the association of modules and experimental conditions for the root samples. Experimental conditions listed in columns and modules in rows. Module eigengene Pearson's correlation coefficient and p-value (in parenthesis) listed for each module per each experimental condition. Cell colors range from high correlation (red) to negative correlation (blue). Number of genes in each module represented by $\mathrm{n}$ in parenthesis after module name. 


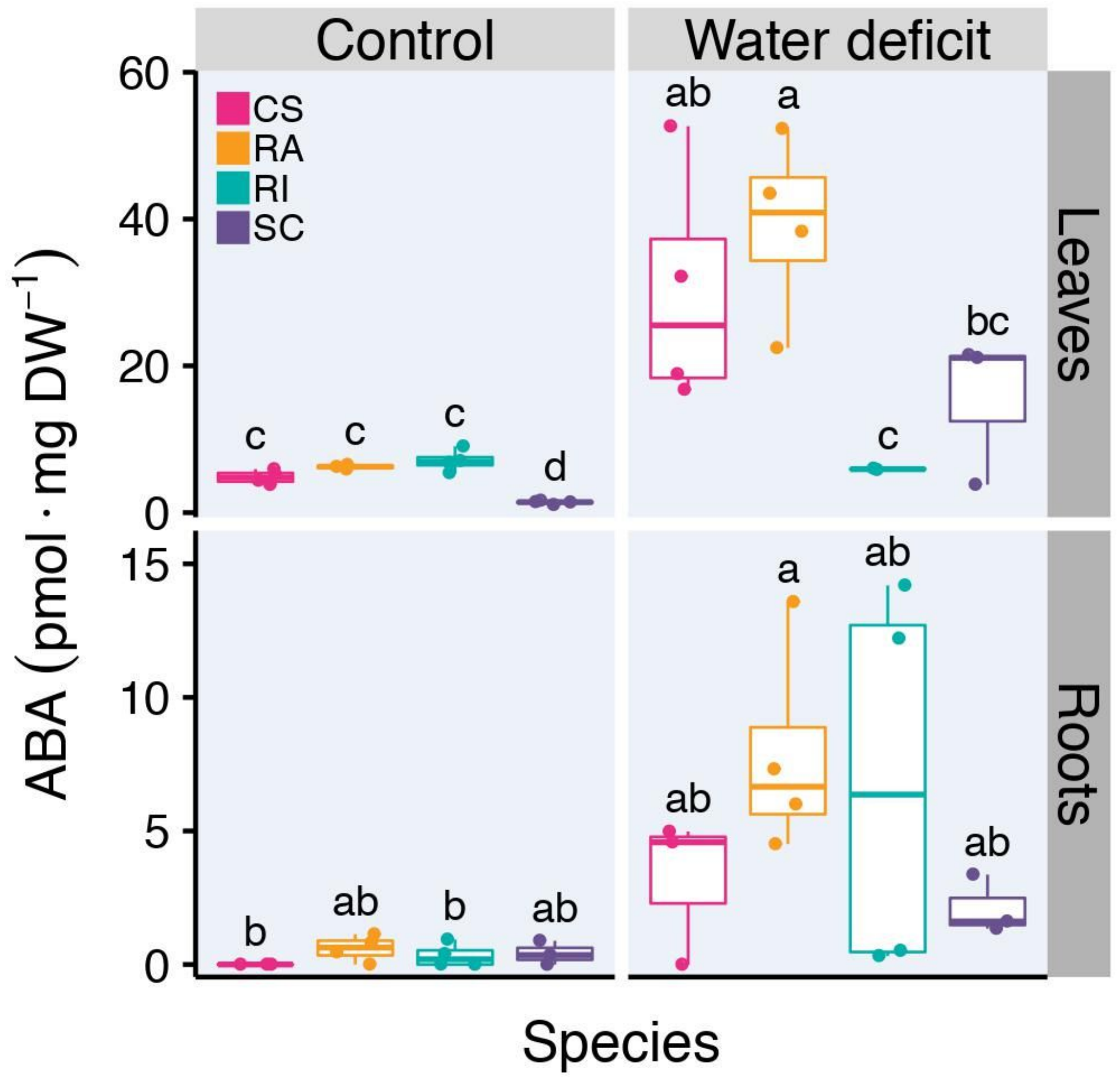

Figure 10

ABA concentrations after one-week of severe WD. ABA concentrations (pmol.mg DW- 1 ) in leaves (top) and roots (bottom) after one-week of Control (left) or WD (right) treatment. Two-way ANOVA Tukey's HSD $(p \leq 0.05)$ letters for each metabolite per organ per week. Pink, orange, blue and purple correspond to CS, $\mathrm{RA}, \mathrm{RI}$, and $\mathrm{SC}$ respectively. Each point represents an individual measurement. $\mathrm{n}=$ three-five individual vines. 
A

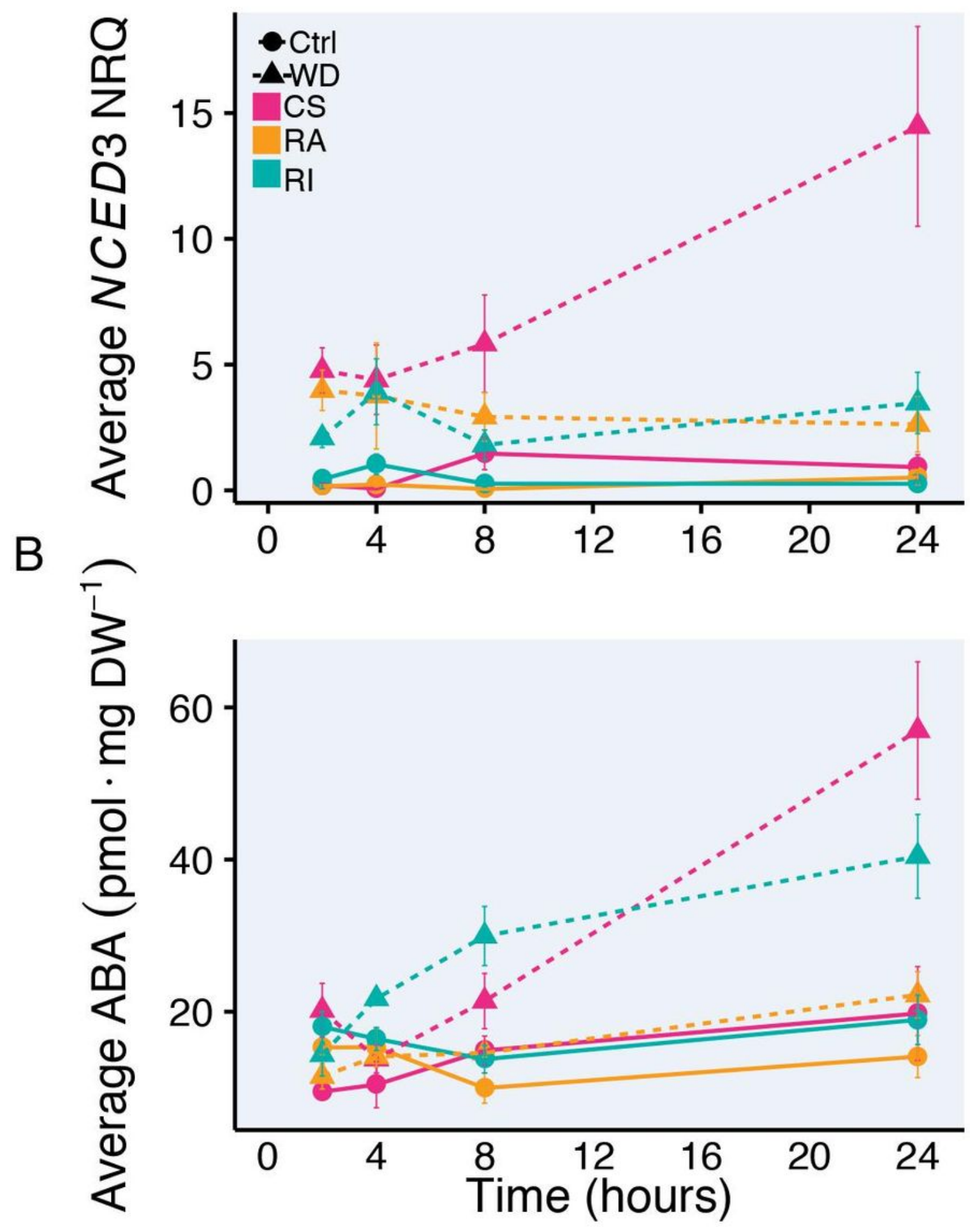

Figure 11

NCED3 transcript NRQ and ABA concentrations after severe rapid dehydration. A) Relative NCED3 transcript abundance (NRQ) and B) ABA concentrations (pmol.mg DW-1) in leaves after 2, 4, 8, and 24 hours of Control (solid line and circles) or rapid dehydration WD (dotted line and triangles) treatment. Two-way ANOVA Tukey's HSD ( $\mathrm{p} \leq \mathrm{0.05}$ ) letters for each metabolite per organ per week. Pink, orange, blue and purple correspond to CS, RA, RI, and SC respectively. Mean $\pm S E, n=$ three-five individual vines. 


\section{Supplementary Files}

This is a list of supplementary files associated with this preprint. Click to download.

- additionalfile1.pdf

- additionalfile2.pdf

- additionalfile3.xlsx

- additionalfile4.xIsx

- additionalfile5.xlsx

- additionalfile6.pdf

- additionalfile7.xlsx

- additionalfile8.xlsx

- additionalfile9.xIsx

- additionalfile10.pdf

- additionalfile11.xlsx

- additionalfile12.xlsx 